\title{
The use of behavioural-science informed interventions to promote latrine use in rural India: a synthesis of findings
}

Charlotte Lane ${ }^{\mathrm{i}}$, International Initiative for Impact Evaluation (3ie)

Sayak Khatua ${ }^{a}$, 3ie

Bethany Caruso $^{\mathrm{b}}$, Emory University

This version: October 20, 2020

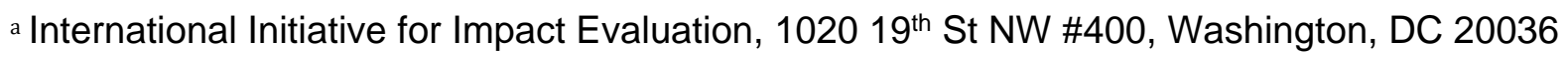
iCorresponding author: clane@3ieimpact.org

b Emory University, 1518 Clifton Road NE, Atlanta, GA 30307 


\section{Abstract}

Background: In 2014, the Indian government launched the Swachh Bharat Mission (SBM) to end open defecation, a historically common practice that has persisted in the country despite household latrine ownership. The majority of SBM resources targeted latrine construction; however, enabling access to sanitation but not guaranteeing use. In 2016, the International Initiative for Impact Evaluation (3ie) funded four clusterrandomized trials to assess the impact of interventions designed to increase latrine use by targeting the behavioral barriers to and facilitators of latrine use among those with household latrines in rural Bihar, Karnataka, Odisha, and Gujarat. The aim of this metasynthesis is to estimate the combined effects of these behavioural-science informed interventions on latrine use in rural India.

Methods: The four trials were conducted by Oxford Policy Management, Swiss Federal Institute of Aquatic Science and Technology (EWAG), Emory University, and the London School of Hygiene and Tropical Medicine. All four trials used an agreed upon set of measures to assess self-reported latrine use, the primary outcome, and a household-level latrine observation index. The use of similar measurements enables comparison. For each study, we calculate an intervention treatment effect through difference-in-differences estimates, which then enables us to calculate the average treatment effect across studies through a difference-in-difference meta-analysis using the individual-participant data.

Results: Self-reported latrine use increased in most states over the course of the intervention ( $\beta_{\text {Bihar }}=0.99[0.57,1.40]$, $\beta_{\text {Karnataka }}=1.24[0.51,1.97]$, $\beta$ odisha $=0.65[0.51,0.80]$, $\left.\beta_{\text {average effect }}=0.66[0.23,1.09], \beta_{\text {Gujarat }}=-0.41[-0.93,0.12]\right)$. The increase tended to be larger in intervention than control villages ( $\left.\beta_{\text {Gujarat }}=0.90[0.12,1.69]\right]$, $\beta_{\text {Karnataka }}=1.05[0.04,0.36]$, $\left.\beta_{\text {odisha }}=0.42[0.19,0.66], \beta_{\text {average effect }}=0.51[0.26,0.76], \beta_{\text {Bihar }}=-0.25[-0.72,0.86]\right)$. However, there was no difference in the change in the latrine observation index between intervention and control villages ( $\beta_{\text {Bihar }}=-0.06[-0.19,0.07]$, $\beta_{\text {Karnataka }}=0.09[-0.17,0.34]$, $\left.\beta_{\text {odisha }}=-0.5[0.15,0.05], \beta_{\text {Gujarat }}=-0.03[-0.31,0.25] \beta_{\text {average effect }}=0.02[-0.06,0.09]\right)$.

Conclusion: We show that the four behavioural-science informed interventions increase self-reported latrine use among those with latrine access beyond increases presumably attributable to SBM alone. As SBM enters its next phase, focusing on the behavioural drivers of latrine use and non-use may help to maintain latrine use behaviors and change the behaviour of those who have yet to start using latrines.

Key words: Behaviour change, open defecation, sanitation, evaluation, WaSH 


\section{Contents}

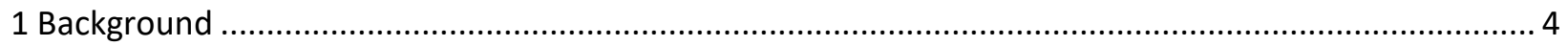

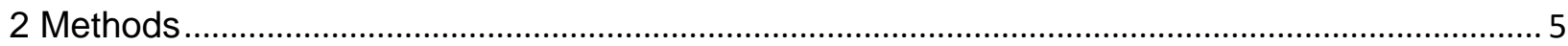

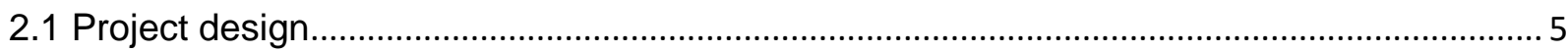

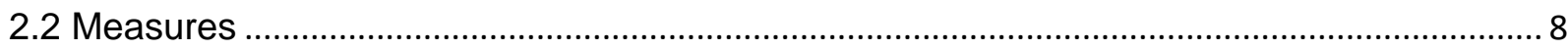

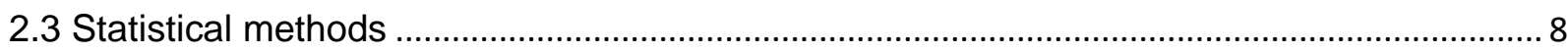

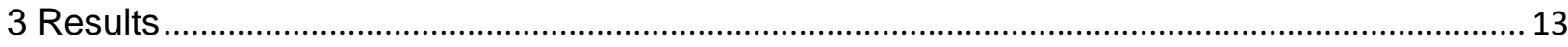

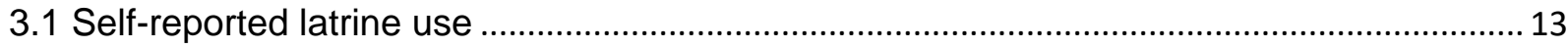

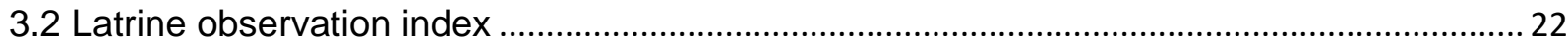

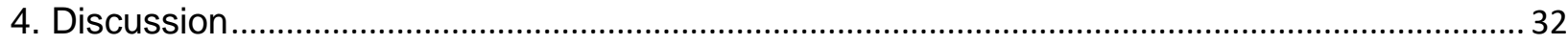

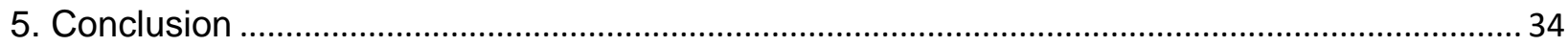

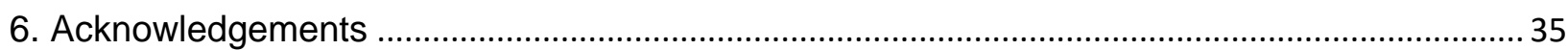

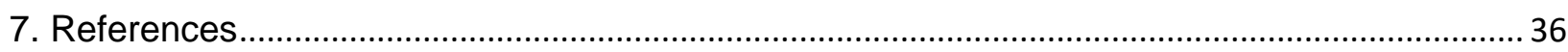

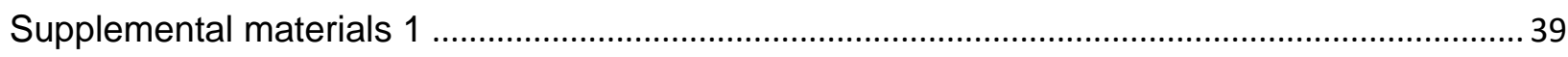

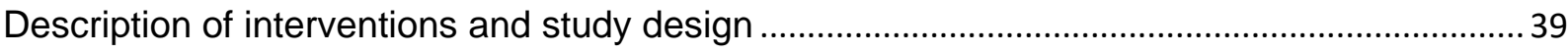

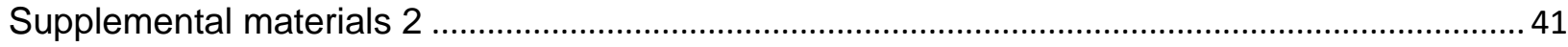

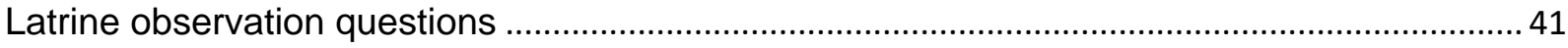

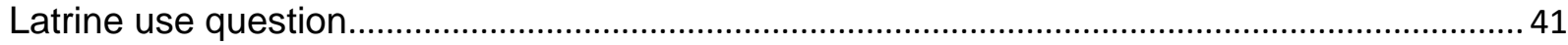

Supplemental materials 3: Quantitative synthesis report pre-analysis plan ............................... 41 


\section{Background}

In 2012, India was the country with the most people practicing open defecation in both absolute (626 million people) and relative (59\% of the total global population) terms (WHO / UNICEF, 2012). To eradicate open defecation, the Indian government launched its flagship program the Swachh Bharat Mission (Clean India Mission; SBM) in 2014 (Department of Drinking Water \& Sanitaiton, 2020). Although India has had a succession of sanitation campaigns, this was the first to have behaviour change, not latrine coverage, as the primary goal. To do this, the SBM focused on large-scale construction of latrines. The Government of India claimed the SBM was responsible for the construction of 10,1968,733 toilets across the country from 2014-2019, reaching 100\% coverage (Department of Drinking Water \& Sanitaiton, 2020).

Although this mass construction is an incredible feat, latrine access does not guarantee latrine use. A 2013 cross-sectional study in Odisha, found that 53\% of those with access to latrines did not use them at all times (Barnard et al., 2013). A 2014 panel study of households with a latrine in Bihar, Madhya Pradesh, Rajasthan and Uttar Pradesh, found that $48 \%$ of households with a working latrine had at least one member practicing open defecation (Coffey et al., 2014). A follow-up study with the same households completed in 2018 found only a slight decrease, with $40 \%$ of latrine-owning households having at least one member defecating in the open (Gupta et al., 2019). Further, they found that the proportion of those who owned a latrine yet defecated in the open remained at 23\% in both 2014 and 2018, demonstrating that the behavior of latrine-owners was not changing (Gupta et al., 2019). As of February 2019, the National Annual Rural Sanitation Survey, which was conducted in the final months of the SBM, concluded that $93 \%$ of households have access to latrines and that $97 \%$ of those with latrine access use them (Agency, 2019), a finding inconsistent with the 2018 follow-up study. The gap between latrine access and latrine use is closing; however, sanitation initiatives must continue to target latrine use behavior if they are to be successful in ending open defecation and sustaining latrine use (Lahiri et al., 2017).

Commonly cited barriers to use in India, in addition to poor latrine quality and the need to fetch water (O'Reilly et al., 2017; Routray et al., 2015), are knowledge, attitudes, and habits (Lahiri et al., 2017; Routray et al., 2015). Open defecation has been viewed as easier and more practical than latrine use. Those who work far from home may not see the need to come home to defecate, especially as this would require the fetching of additional water (O'Reilly et al., 2017; Routray et al., 2015). In addition, women perceive open defecation as a social opportunity to leave their house and meet with friends (Routray et al., 2015). Deep-rooted concepts of ritualistic purity further the attitude that latrine use is undesirable (Coffey et al., 2017; Juran et al., 2019; Routray et al., 2015). Many people, especially those of higher castes, are concerned with latrine pits filling quickly and requiring emptying (Coffey et al., 2017; Lahiri et al., 2017; O'Reilly et al., 2017; Routray et al., 2015). People of higher castes may avoid using a latrine so as to also avoid having to engage with low caste individuals to empty the pit or to do so themselves (Coffey et al., 2017; Routray et al., 2015). There is variation in the perceived 
convenience of pit emptying, with some people claiming no challenges in pit emptying and others, especially in remote villages, having trouble finding workers to empty pits (O'Reilly et al., 2017; Routray et al., 2015).

Launched in 2016, the International Initiative for Impact Evaluation's (3ie) Promoting Latrine Use in Rural India evidence program funded teams to use insights from behavioural science to design, pilot, and scale low-cost, context-specific interventions to increase latrine use. During the first phase of the evidence program, nine teams designed and piloted context-specific interventions in eight Indian states over a period of three months. In the next phase, 3ie funded four of the nine teams to conduct impact evaluations of the most promising interventions. The appraisal of the relative promise of the selected teams and their interventions was conducted by a set of five independent reviewers, who considered scalability, cost effectiveness, and expected impact on outcomes. Here, we present the individual and combined effects from these four impact evaluations.

We focus on the estimation of the average treatment effect achieved by the four studies. Policy makers can consider this average treatment effect when designing their own sanitation programs as it is likely to be more generalizable than the results of each separate study. Because all four studies used the same set of questions to evaluate change in latrine use and we analyze the individual participant data to calculate estimates, our combined analysis constitutes a gold standard, prospective, individualparticipant, meta-analysis (Ioannidis, 2017). The prospective nature of this work reduces bias, while the use of individual participant data increases precision and allows for the harmonization of the data and analyses. We hope that researchers consider the benefits of the collaborative research design employed here in the development of future programs that require significant cross-context data in order to draw strong conclusions.

\section{Methods}

\subsection{Project design}

Four teams, from Oxford Policy Management (OPM), Swiss Federal Institute of Aquatic Science and Technology (EWAG), Emory University, and the London School of Hygiene and Tropical Medicine (LSHTM), designed the most promising interventions that are reported on here and conducted impact evaluations of those interventions over 18 months in the states of Bihar, Karnataka, Odisha, and Gujarat respectively. Each evaluation -all cluster-randomized trials-assessed interventions that were designed to target the site-specific barriers to latrine use in the manner that the study teams believed would be most effective (Table 1). All studies randomized delivery of the interventions at the Gram Panchayat or village level and included between 66 and 120 clusters. Control sites were similar in basic sociodemographic characteristics (Table 2; Caruso et al., 2019b, 2019a; Chuahan et al., 2019; Friedrich et al., 2019; International Initiative for Impact Evaluation, 2020; Viswanathan et al., 2019). Both control and intervention sites received SBM activities. Each team chose their own target populations 
based on local context, including the availability of different types of latrines and open defecation rates. Additional detail regarding project activities, sampling design, and data collection is provided elsewhere (Supplemental materials 1; Caruso et al., 2019b, 2019a; Chuahan et al., 2019; Friedrich et al., 2019; International Initiative for Impact Evaluation, 2020; Viswanathan et al., 2019).

All four studies collected data pre- and post-intervention. Three of the four studies collected data on the same households at baseline and endline. The fourth, which took place in Gujarat, used a repeated cross sectional design, interviewing different households at baseline and endline due to concern about respondent fatigue (Schmidt, Wolf-peter, Chauhan et al., 2020). 
Table 1: Summary of the four studies conducted to promote latrine use among those with latrine access in rural India.

\begin{tabular}{|c|c|c|c|c|c|c|c|}
\hline $\begin{array}{l}\text { State (Lead } \\
\text { research } \\
\text { team, } \\
\text { implementing } \\
\text { partner) }\end{array}$ & Eligibility criteria & Districts & Villages & $\begin{array}{l}\text { Behavioural } \\
\text { science } \\
\text { approach }\end{array}$ & Activities & $\begin{array}{l}\text { Number of } \\
\text { individuals } \\
\text { (households) } \\
\text { sampled at } \\
\text { baseline }\end{array}$ & $\begin{array}{l}\text { Number of } \\
\text { individuals } \\
\text { (households) } \\
\text { sampled at } \\
\text { endline }\end{array}$ \\
\hline $\begin{array}{l}\text { Bihar (OPML, } \\
\text { World Vision } \\
\text { India) }\end{array}$ & $\begin{array}{l}\text { Households with } \\
\text { functional twin pit } \\
\text { latrines }\end{array}$ & $\begin{array}{l}\text { Jamui, } \\
\text { Khagaria, } \\
\text { Madhepura, } \\
\text { Nalanda, } \\
\text { Nawada and } \\
\text { Saharsa }\end{array}$ & $\begin{array}{l}86 \text { villages } \\
\text { (28 ODF } \\
\text { villages) }\end{array}$ & Nudge theory & $\begin{array}{l}\text { Card game, pledge, } \\
\text { calendar, lockbox, } \\
\text { French drain } \\
\text { demonstration and } \\
\text { posters to support } \\
\text { latrine use }\end{array}$ & $6,158(1,107)$ & $6,046(1,085)$ \\
\hline $\begin{array}{l}\text { Karnataka } \\
\text { (EWAG, } \\
\text { Water Aid } \\
\text { India) }\end{array}$ & $\begin{array}{l}\text { Households with } \\
\text { functional latrines }\end{array}$ & Raichur & $\begin{array}{l}120 \text { villages } \\
\text { (No ODF } \\
\text { villages) }\end{array}$ & $\begin{array}{l}\text { Risks, attitudes, } \\
\text { norms, abilities } \\
\text { and self- } \\
\text { regulation } \\
\text { (RANAS) }\end{array}$ & $\begin{array}{l}\text { Community, } \\
\text { household, and } \\
\text { mothers groups } \\
\text { meetings and a } \\
\text { supportive phone } \\
\text { call }\end{array}$ & $10793(2,185)$ & $10656(2,021)$ \\
\hline $\begin{array}{l}\text { Gujarat } \\
\text { (LSHTM, } \\
\text { IIPHG2) }\end{array}$ & $\begin{array}{l}\text { Households with } \\
\text { functional } \\
\text { government or } \\
\text { contractor built } \\
\text { latrines }\end{array}$ & Bhavnagar & $\begin{array}{l}90 \text { villages } \\
\text { (All ODF } \\
\text { villages) }\end{array}$ & $\begin{array}{l}\text { Behaviour center } \\
\text { design }\end{array}$ & $\begin{array}{l}\text { Social marketing } \\
\text { campaign to support } \\
\text { the use of a five star } \\
\text { toilet }\end{array}$ & 3496 (696) & $13960(2,492)$ \\
\hline $\begin{array}{l}\text { Odisha } \\
\text { (Emory, Rural } \\
\text { Welfare } \\
\text { Institute) }\end{array}$ & $\begin{array}{l}\text { Households with } \\
\text { latrines }\end{array}$ & Puri & $\begin{array}{l}\text { (6 ODF } \\
\text { villages) }\end{array}$ & $\begin{array}{l}\text { Risk perceptions, } \\
\text { abilities, social } \\
\text { norms, } \\
\text { motivation, } \\
\text { physical } \\
\text { opportunity, and } \\
\text { self-regulation }\end{array}$ & $\begin{array}{l}\text { Palla, transect walk, } \\
\text { wall painting, } \\
\text { household visits, } \\
\text { latrine repairs, } \\
\text { community and } \\
\text { mothers meetings, } \\
\text { and positive deviant } \\
\text { recognition }\end{array}$ & $16827(3,723)$ & $16827(3,723)$ \\
\hline
\end{tabular}

1. The same participants were not followed at baseline and endline 


\subsection{Measures}

All four studies collected data using their own surveys and a standardized set of indicators that were common across all four teams. The four teams developed these indicators through an intensive, collaborative process. The studies used the same measures to facilitate an accurate and comparable assessment of behavior change. All four study teams collected data on latrine type, size of the pit, and demographics of the participants (Table 2).

The primary outcome of interest was self-reported latrine use. To determine selfreported latrine use, information about where each household member last defecated was queried for each household member aged five years or older (Supplemental materials 2).

To determine if the latrine was actually in use, enumerators on each team observed and recorded data regarding the state of the household latrine, including if the pan was clogged, water was available, and the latrine appeared to be in use. These data were used to create a latrine use observation index. The latrine use observation index score was calculated by assigning a value of zero or one to each of the five primary latrine observation questions (Supplemental materials 2) and summing these values. The index score reflects the number of characteristic signs of latrine use observed in the household latrine, not the individual's behaviour itself. A value of zero reflects that none of the characteristic signs of latrine use were present while a value of five indicates that all characteristic signs of latrine use were seen. The mean value of this index before the intervention was 1.78 and fell slightly to 1.73 after the intervention.

\subsection{Statistical methods}

We use a difference-in-difference approach with clustered standard errors to estimate the impact of each study and allow for comparisons of the effects between studies (equation 1). Difference-in-differences were calculated within each of the four studies to allow for the comparison of results across studies as each team had previously conducted analysis using methods that were not comparable. The difference-indifference was then calculated across the four studies for a prospective, individualparticipant, meta-analysis. For each individual study site, a regression with robust standard errors clustered at the village level was used to evaluate the effect of the intervention (Bottomley et al., 2016). To calculate the combined effect, a separate regression was conducted across all four datasets, with robust standard errors clustered at the state level and fixed effects for state. Robust standard errors were preferred to other methods to account for clustering, such as multilevel modeling or generalized estimating equations, because they make fewer distributional assumptions. Calculations were done based on equation one in $\mathrm{R}$ using the mice and miceadds packages (Robitzsch and Grund, 2020; van Buuren and Grothuis-Oubshoorn, 2011).

$$
\text { Equation 1: Outcome } \left.=\mathrm{A}+\beta_{1} \text { (endline }\right)+\beta_{2}(\text { treatment })+\beta_{3}(\text { endline })(\text { treatment })+\mathrm{C}+\varepsilon
$$


In equation one, $\beta_{1}$ represents the change in control villages between baseline and endline and $\beta_{3}$ the difference in the change between control and intervention villages. This is the difference-in-difference term and reflects the log of the odds ratio. $C$ represents the matrix of controls. The control variables were sex, age, household size, caste, and religion. Age and household size were standardized across the four studies so that the order of magnitude of these variables was consistent. The control variables were chosen because they are not balanced between intervention and control arms at baseline in many of the study sites and / or the literature indicates that they are likely to affect latrine use (Routray et al.,2015; Sinha et al., 2017).

Two sets of analysis were conducted. One set of analysis considered self-reported latrine use as the outcome and was calculated with a logit. A second set of analysis was conducted using the latrine observation index as the outcome of interest and was calculated with a standard regression. The value of the household latrine use observation index was applied to each person within the same household. This approach, rather than considering only one value per household, was preferred because it allows for households to be weighted by their size. Larger households have a proportionately larger effect in this analysis. Coefficients reflect the relationship between an individual's characteristics and the number of characteristics signs of latrine use seen in that person's household latrine. The approach was specified in a pre-analysis plan, with some modifications (Supplemental materials 3).

Although repeated cross sectional designs are common in difference-in-difference analysis (Models et al., 2011), several sensitivity analyses were employed due to the unique design of the Gujarat study. The difference-in-difference was first calculated according to equation one, as with the other studies. Because some villages were only sampled after the intervention, a sensitivity analysis was conducted using only villages sampled at both periods. In an additional sensitivity analysis, multiple imputation was conducted in which the pre-intervention dataset was used to impute the pre-intervention values of the individuals interviewed after the intervention and the difference-indifference was calculated on the imputed datasets (Robitzsch and Grund, 2020; van Buuren and Grothuis-Oubshoorn, 2011). Age, sex, caste, religion, household size, village, and number of pits in the latrine were used to impute values, allowing for clustering at the village level. A level-1 logistic, generalized linear mixed effects model was used to impute the binary value for self-reported latrine use. A random forest model was used to impute the integer value for the latrine observation index. The imputation was run using 100 iterations.

Because of the different approaches used to estimate the effect of the intervention in Gujarat, sensitivity analyses were also used in the estimation of the average treatment effect. In addition to being calculated across the whole dataset, the average effect was estimated within the three states which sampled the same households at baseline and endline and using the imputed data. 
Table 2: Characteristics of study participants by study arm and period. Presented as counts and means.

Bihar

Villages

Households

Children

Adults3

Women

Age of

adults4

Castes

General

category

OBC

$\mathrm{SC}$

ST

Minority I

other

Not

reported

Religion

Hindu

Muslim

Christian

Other

Household

has a

single pit

latrine5

Report that the latrine

has filled5

Latrine

observation

index

Self-report

latrine use

Report the

safe

disposal of

child faeces

Karnataka

\begin{tabular}{cccccc}
\multicolumn{2}{c}{ Pre-intervention } & & \multicolumn{2}{c}{ Post-intervention } & $\begin{array}{c}\text { P- } \\
\text { Control Intervention }\end{array} \begin{array}{c}\mathrm{P}- \\
\text { value1 }\end{array}$
\end{tabular}

$64 \quad 60$

$557 \quad 550$

$434 \quad 456$

2588

1323

28.337

169

2196

581

70

0

7

2734

277

12

0

0

20

1.318

2120

59

59

336

811

56

0

22

3016

109

0

10

0

\section{6}

1338

29.283

1910

$$
<0.001
$$

$<0.001$

$<0.001$

63

551

419

2639

1346

28.987

0.001

$--$

0.009

193

2060

707

87

0

12

$<0.001$

$<0.001$

0.001

$--$

$34<0.001$

41

2767
285

7

0

0

$\begin{array}{ll}1.27 & 0.017\end{array}$

1.555

1.47

2881

106

0

0

306

1902

736

43

0

0

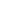

0

45

0.162

$<0.001$

0.133

0.344

0.023

0.886

0.359

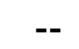

2421

2336

0.018

0.113

2183

0.984

0.265

57

61

0.522

0.201 


\begin{tabular}{|c|c|c|c|c|c|c|c|}
\hline Villages & 54 & 56 & & 54 & 56 & & \\
\hline Households & 1087 & 1098 & & 1010 & 1011 & & \\
\hline Children & 361 & 367 & 0.719 & 370 & 350 & 0.732 & 1.000 \\
\hline Adults6 & 5066 & 4996 & 0.748 & 5033 & 4903 & 0.732 & 1.000 \\
\hline Women & 2513 & 2471 & 0.911 & 2483 & 2421 & 0.970 & 0.951 \\
\hline $\begin{array}{l}\text { Age of } \\
\text { adults } 4\end{array}$ & 30.671 & 31.460 & 0.018 & 30.235 & 31.073 & 0.012 & 0.001 \\
\hline \multicolumn{8}{|l|}{ Castes } \\
\hline $\begin{array}{l}\text { General } \\
\text { category }\end{array}$ & 872 & 801 & $<0.001$ & 876 & 784 & $<0.001$ & 0.010 \\
\hline OBC & 2056 & 1794 & $<0.001$ & 2056 & 1755 & $<0.001$ & 0.045 \\
\hline SC & 872 & 1255 & $<0.001$ & 839 & 1235 & $<0.001$ & 0.004 \\
\hline ST & 1264 & 1287 & 0.292 & 1285 & 1252 & 0.068 & 0.036 \\
\hline $\begin{array}{l}\text { Minority I } \\
\text { other }\end{array}$ & 296 & 218 & 0.000 & 294 & 219 & 0.000 & 0.000 \\
\hline $\begin{array}{l}\text { Not } \\
\text { reported }\end{array}$ & 69 & 9 & 0.000 & 53 & 8 & 0.000 & 0.175 \\
\hline \multicolumn{8}{|l|}{ Religion } \\
\hline Hindu & 4865 & 4917 & 0.613 & 4850 & 4816 & 0.714 & 0.394 \\
\hline Muslim & 346 & 228 & $<0.001$ & 342 & 246 & $<0.001$ & 0.792 \\
\hline Christian & 117 & 34 & $<0.001$ & 106 & 14 & $<0.001$ & 0.040 \\
\hline $\begin{array}{l}\text { Other } \\
\text { Household }\end{array}$ & 101 & 185 & $<0.001$ & 105 & 177 & $<0.001$ & 0.706 \\
\hline $\begin{array}{l}\text { has a } \\
\text { single pit } \\
\text { latrine5 }\end{array}$ & 1023 & 1060 & 0.508 & 950 & 976 & 0.897 & 0.508 \\
\hline $\begin{array}{l}\text { Report that } \\
\text { the latrine } \\
\text { has filled5 }\end{array}$ & 26 & 18 & 0.000 & 23 & 21 & 0.012 & 0.000 \\
\hline $\begin{array}{l}\text { Latrine } \\
\text { observation } \\
\text { index }\end{array}$ & 2.338 & 2.33 & 0.415 & 2.343 & 2.45 & $<0.001$ & 0.049 \\
\hline $\begin{array}{l}\text { Self-report } \\
\text { latrine use }\end{array}$ & 3788 & 3704 & 0.390 & 4532 & 4674 & $<0.001$ & $<0.001$ \\
\hline $\begin{array}{l}\text { Report the } \\
\text { safe } \\
\text { disposal of } \\
\text { child faeces } \\
\text { Gujarat }\end{array}$ & 24 & 20 & 0.553 & 139 & 93 & 0.007 & 0.003 \\
\hline $\begin{array}{l}\text { Number of } \\
\text { villages }\end{array}$ & 40 & 39 & & 47 & 47 & & \\
\hline $\begin{array}{l}\text { Number of } \\
\text { households }\end{array}$ & 366 & 330 & & 1214 & 1278 & & \\
\hline Children & 106 & 101 & 0.725 & 492 & 555 & 0.492 & 0.359 \\
\hline Adults & 1734 & 1555 & 0.725 & 6217 & 6696 & 0.492 & 0.359 \\
\hline Women & 897 & 802 & 0.938 & 3137 & 3355 & 0.701 & 0.664 \\
\hline
\end{tabular}




\begin{tabular}{|c|c|c|c|c|c|c|c|}
\hline $\begin{array}{l}\text { Age of } \\
\text { adults } 4\end{array}$ & 32.746 & 33.143 & 0.554 & 33.133 & 33.796 & 0.054 & 0.041 \\
\hline \multicolumn{8}{|l|}{ Castes } \\
\hline $\begin{array}{l}\text { General } \\
\text { category }\end{array}$ & 185 & 132 & 0.003 & 269 & 181 & $<0.001$ & $<0.001$ \\
\hline $\mathrm{OBC}$ & 19 & 22 & 0.755 & 27 & 18 & $<0.001$ & $<0.001$ \\
\hline SC & 267 & 196 & 0.001 & 1529 & 1822 & $<0.001$ & $<0.001$ \\
\hline ST & 1344 & 1294 & 0.340 & 3848 & 4253 & $<0.001$ & $<0.001$ \\
\hline $\begin{array}{l}\text { Minority I } \\
\text { other }\end{array}$ & 25 & 12 & 0.049 & 544 & 422 & $<0.001$ & 0.010 \\
\hline $\begin{array}{l}\text { Not } \\
\text { reported }\end{array}$ & 0 & 0 & -- & 492 & 555 & 0.055 & $<0.001$ \\
\hline \multicolumn{8}{|l|}{ Religion } \\
\hline Hindu & 1806 & 1615 & 0.0012 & 6138 & 6611 & $<0.001$ & $<0.001$ \\
\hline Muslim & 34 & 41 & 0.4884 & 79 & 85 & $<0.001$ & -- \\
\hline Christian & 0 & 0 & -- & 0 & 0 & -- & -- \\
\hline Other & 0 & 0 & -- & 492 & 555 & $<0.001$ & -- \\
\hline $\begin{array}{l}\text { Household } \\
\text { has a }\end{array}$ & & & & & & & \\
\hline $\begin{array}{l}\text { single pit } \\
\text { latrine5 }\end{array}$ & 312 & 291 & 0.579 & 33 & 39 & 0.256 & 0.008 \\
\hline $\begin{array}{l}\text { Report that } \\
\text { the latrine } \\
\text { has filled } 5\end{array}$ & 3.189 & 3.306 & 0.001 & 2.082 & 2.155 & $<0.001$ & $<0.001$ \\
\hline $\begin{array}{l}\text { Report } \\
\text { latrine use }\end{array}$ & 1543 & 1313 & 0.004 & 5255 & 6089 & $<0.001$ & $<0.001$ \\
\hline $\begin{array}{l}\text { Latrine } \\
\text { observation } \\
\text { index }\end{array}$ & & & & & & & $<0.002$ \\
\hline $\begin{array}{l}\text { index } \\
\text { Report the }\end{array}$ & 3.189 & 3.306 & 0.001 & 2.082 & 2.155 & 0.000 & \\
\hline $\begin{array}{l}\text { safe } \\
\text { disposal of } \\
\text { child faeces } \\
\text { Odisha }\end{array}$ & 33 & 19 & 0.051 & 44 & 52 & 1.000 & 0.175 \\
\hline Villages & 33 & 33 & & 33 & 33 & & \\
\hline Households & 1916 & 1807 & & 1916 & 1807 & & \\
\hline Children & 581 & 579 & 0.544 & 581 & 579 & 0.544 & 0.379 \\
\hline Adults & 7999 & 7668 & 0.544 & 8001 & 7670 & 0.544 & 0.379 \\
\hline Women & 4041 & 3822 & 0.399 & 4043 & 3824 & 0.385 & 0.229 \\
\hline $\begin{array}{l}\text { Age of } \\
\text { adults } 4\end{array}$ & 37.221 & 37.335 & 0.716 & 37.218 & 37.339 & 0.699 & 0.595 \\
\hline \multicolumn{8}{|l|}{ Castes } \\
\hline $\begin{array}{l}\text { General } \\
\text { category }\end{array}$ & 6533 & 6055 & $<0.001$ & 6535 & 6057 & $<0.001$ & $<0.001$ \\
\hline OBC & 52 & 112 & $<0.001$ & 52 & 112 & $<0.001$ & $<0.001$ \\
\hline SC & 965 & 1152 & $<0.001$ & 965 & 1152 & $<0.001$ & $<0.001$ \\
\hline
\end{tabular}




\begin{tabular}{|c|c|c|c|c|c|c|c|}
\hline ST & 0 & 0 & -- & 0 & 0 & -- & -- \\
\hline $\begin{array}{l}\text { Minority I } \\
\text { other }\end{array}$ & 678 & 654 & $<0.001$ & 678 & 654 & $<0.001$ & $<0.001$ \\
\hline $\begin{array}{l}\text { Not } \\
\text { reported }\end{array}$ & 352 & 274 & 0.002 & 352 & 274 & 0.002 & 1.000 \\
\hline \multicolumn{8}{|l|}{ Religion } \\
\hline Hindu & 8127 & 7955 & 0.149 & 8129 & 7957 & 0.149 & 0.916 \\
\hline Muslim & 336 & 158 & $<0.001$ & 336 & 158 & $<0.001$ & 0.470 \\
\hline Christian & 0 & 2 & -- & 0 & -- & 0.016 & $<0.001$ \\
\hline Other & 117 & 132 & 0.899 & 117 & 132 & 0.899 & 0.303 \\
\hline Household & & & & & & & \\
\hline $\begin{array}{l}\text { has a } \\
\text { single pit } \\
\text { latrine5 }\end{array}$ & 79 & 1807 & 0.595 & 0 & 1807 & $<0.001$ & $<0.001$ \\
\hline $\begin{array}{l}\text { Report that } \\
\text { the latrine } \\
\text { has filled } 5\end{array}$ & 85 & 79 & 0.633 & 83 & 82 & 0.462 & 0.398 \\
\hline $\begin{array}{l}\text { Latrine } \\
\text { observation } \\
\text { index }\end{array}$ & 1.246 & 1.278 & 0.075 & 1.121 & 1.085 & 0.181 & 0.840 \\
\hline $\begin{array}{l}\text { Self-report } \\
\text { latrine use }\end{array}$ & 4756 & 4464 & 0.094 & 5767 & 5976 & $<0.001$ & $<0.001$ \\
\hline $\begin{array}{l}\text { Report the } \\
\text { safe } \\
\text { disposal of } \\
\text { child faeces }\end{array}$ & 14 & 34 & 0.006 & 34 & 101 & $<0.001$ & $<0.001$ \\
\hline
\end{tabular}

1. P-values were calculated as z-tests and t-tests as appropriate, comparing values between intervention and control arms within each period.

2. P-values were calculated at ANOVA and chi-square as appropriate, comparing values across time and treatment status.

3. Age missing in 3 individuals who are therefore coded as neither adults nor children

4. Age and latrine observation indexes are presented as means, not counts. P-values reflect t-tests and ANOVAs.

5. Reported at the household level

6. Age missing in 6 individuals who are therefore coded as neither adults nor children

Acronyms: OBC - other backward caste, SC - schedule caste, ST - schedule tribe

\section{Results}

\subsection{Self-reported latrine use}

\subsubsection{Univariate analysis}

Self-reported latrine use increased in control villages in Bihar, Karnataka, and Odisha over the course of the intervention, resulting in an average increase in self-reported latrine use in control villages (Table 3, $\beta_{\text {Bihar }}=0.93[0.51,1.36]$, $\beta_{\text {Karnataka }}=1.19[0.48,1.89]$, $\left.\beta_{\text {odisha }}=0.164[0.49,0.74], \beta_{\text {Average effect }}=0.70[0.48,0.91]\right)$. However, there was no change in self-reported latrine use in control villages in Gujarat $(\beta=-0.44[-0.97,0.10])$. In 
Karnataka, Odisha, and Gujarat, there were larger increases in self-reported latrine use in the intervention sites than the control sites $\left(\beta_{\text {Karnataka }}=1.03[0.05,2.02]\right.$,

$\left.\beta_{\text {odisha }}=0.41[0.19,0.62], \beta_{\text {Gujarat }}=0.92[0.15,1.70]\right)$. Similarly, the average treatment effect indicated that self-reported latrine use increased more in intervention sites than control sites across the four studies $(\beta=0.49[0.23,0.75])$. However, there was no difference in the change in latrine use between intervention and control sites in Bihar $(\beta=-0.24[-$ $0.72,0.25])$.

Table 3: Unadjusted effect estimates of the intervention on self-reported latrine use

\begin{tabular}{lccc}
\hline Bihar $(\mathbf{n}=\mathbf{1 0 3 9 8})$ & Coefficient & $\begin{array}{c}\text { P- } \\
\text { value }\end{array}$ & $\mathbf{C l}$ \\
Period1 & 0.934 & 0.000 & $0.5096,1.3574$ \\
Arm2 & -0.004 & 0.992 & $-0.775,0.7671$ \\
Treatment by period interaction & -0.236 & 0.336 & $-0.717,0.2450$ \\
Karnataka ( $\mathbf{n = 1 9 8 8 1 )}$ & & & \\
Period & 1.180 & 0.001 & $0.4759,1.8850$ \\
Arm & -0.041 & 0.826 & $-0.402,0.3215$ \\
Treatment by period interaction & 1.033 & 0.039 & $0.0498,2.0160$ \\
Odisha $(\mathbf{n}=\mathbf{3 0 4 0 7 )}$ & & & \\
Period & 0.614 & 0.000 & $0.4878,0.7402$ \\
Arm & -0.056 & 0.747 & $-0.396,0.2845$ \\
Treatment by period interaction & 0.406 & 0.000 & $0.1932,0.6182$ \\
Gujarat ( $\mathbf{n}=16163)$ & & & \\
Period & -0.440 & 0.107 & $-0.974,0.0956$ \\
Arm & -0.314 & 0.324 & $-0.938,0.3102$ \\
Treatment by period interaction & 0.922 & 0.020 & $0.1466,1.6969$ \\
Meta-analysis ( $\mathbf{n}=76849)$ & & & \\
Period & 0.695 & 0.000 & $0.4792,0.9099$ \\
Arm & -0.055 & 0.042 & $-0.107,-0.001$ \\
Treatment by period interaction & 0.490 & 0.000 & $0.2302,0.7506$ \\
\hline
\end{tabular}

1. Coded as 0 for pre-intervention and 1 for post-intervention

2. Coded as 0 for control and 1 for treatment

Table 3: Unadjusted effect estimates of the intervention on self-reported latrine use

\begin{tabular}{|c|c|c|c|}
\hline Bihar $(n=10398)$ & Coefficient & $\begin{array}{c}\mathrm{P}- \\
\text { value }\end{array}$ & $\mathrm{Cl}$ \\
\hline Period $^{1}$ & 0.934 & 0.000 & $0.5096,1.3574$ \\
\hline $\mathrm{Arm}^{2}$ & -0.004 & 0.992 & $-0.775,0.7671$ \\
\hline $\begin{array}{l}\text { Treatment by period interaction } \\
\text { Karnataka }(n=19881)\end{array}$ & -0.236 & 0.336 & $-0.717,0.2450$ \\
\hline Period & 1.180 & 0.001 & $0.4759,1.8850$ \\
\hline
\end{tabular}




\begin{tabular}{lccc} 
Arm & -0.041 & 0.826 & $-0.402,0.3215$ \\
Treatment by period interaction & 1.033 & 0.039 & $0.0498,2.0160$ \\
Odisha $(\mathbf{n = 3 0 4 0 7 )}$ & & & \\
Period & 0.614 & 0.000 & $0.4878,0.7402$ \\
Arm & -0.056 & 0.747 & $-0.396,0.2845$ \\
Treatment by period interaction & 0.406 & 0.000 & $0.1932,0.6182$ \\
Gujarat $(\mathbf{n = 1 6 1 6 3 )}$ & & & \\
Period & -0.440 & 0.107 & $-0.974,0.0956$ \\
Arm & -0.314 & 0.324 & $-0.938,0.3102$ \\
Treatment by period interaction & 0.922 & 0.020 & $0.1466,1.6969$ \\
Meta-analysis ( $\mathbf{n = 7 6 8 4 9 )}$ & & & \\
Period & 0.695 & 0.000 & $0.4792,0.9099$ \\
Arm & -0.055 & 0.042 & $-0.107,-0.001$ \\
Treatment by period interaction & 0.490 & 0.000 & $0.2302,0.7506$ \\
\hline
\end{tabular}

1. Coded as 0 for pre-intervention and 1 for post-intervention

2. Coded as 0 for control and 1 for treatment

\subsubsection{Multivariate analysis}

Self-reported latrine use increased in control arms across three sites during the intervention, with a no change in Gujarat (Table 4, $\beta_{\text {Bihar }}=0.99[0.57,1.40]$, $\beta_{\text {Karnataka }}=1.24[0.51,1.97]$, $\beta_{\text {Odisha }}=0.65[0.51,0.80], \beta_{\text {Average effect }}=0.66[0.23,1.09]$, $\beta_{\text {Gujarat }}=-$ $0.41[-0.93,0.12])$. There was a larger increase in latrine use in the intervention sites than the control sites in Karnataka, Odisha, and Gujarat (Figure 1, $\beta_{\text {karnataka }}=1.05[0.04,2.06]$, $\beta_{\text {odisha }}=0.42[0.19,0.66], \beta$ Gujarat $\left.=0.90[0.12,1.69]\right)$, with no changes in $\operatorname{Bihar}(\beta=-0.25[-$ $0.72,0.86])$. On average, intervention sites had larger increases in latrine use than control sites as well ( $\left.\beta_{\text {Average effect }}=0.51[0.26,0.76]\right)$.

Women tended to self-report latrine use more than men ( $\beta_{\text {Bihar }}=0.42[0.30 .0 .53]$, $\beta_{\text {Karnataka }}=0.54[0.43,0.66], \beta_{\text {odisha }}=0.51[0.45,0.57], \beta_{\text {Gujarat }}=0.01[-0.08,0.10], \beta_{\text {Average }}$ effect $=0.42[0.24,0.61])$. Age had a differing relationship with latrine use across the four sites. In Odisha, older individuals were less likely to report use (ßodisha $=-0.01[-0.01,0]$ ). In Karnataka and Gujarat, older individuals were more likely to report use $\left(\right.$ karnataka $=0.031\left[0.03[0.02,0.04], \beta_{\text {Gujarat }}=0.02[0,0.03]\right)$. Caste had variable relationships with latrine use in the four studies. However, in general and in the meta-analysis, schedule casts had the highest reported latrine use.

In the meta-difference-in-difference, individuals from Karnataka and Odisha had lower self-reported latrine use than those from Bihar ( $\beta_{\text {Karnataka }}=-0.15[-0.20 .-0.10]$, ßodisha $=-$ $1.61[-1.77,-1.43])$. Those from Gujarat had higher self-reported latrine use than those from Bihar $(\beta=0.18[0.08,0.28])$. 


\subsubsection{Multivariate sensitivity analysis}

The results of the analysis in Gujarat changed somewhat under alternate sample specifications (Supplemental table 2, Supplemental figure 1). When the sample was restricted to villages which were measured before and after the intervention, the primary findings were the same: latrine use did not increase significantly across time in control sites $(\beta=-0.47[-1.00,0.70])$, but there was a significant difference in the change in latrine use between intervention and control arms $(\beta=1.03[0.23,1.84])$. Latrine use still increased with age $(\beta=0.01[0.00,0.02])$. And the tendency of women to use latrines more than men remained non-significant $(\beta=0.02[-0.07,0.11])$. When the preintervention sample in Gujarat was used to impute the baseline latrine use of the postintervention sample, latrine use did not increase across time $(\beta=-0.19[-1.02,0.64])$. The treatment effect became non-significant, but stayed in the same direction $(\beta=0.66[-$ $0.29,1.62])$.

Results did not change meaningfully when Gujarat was excluded from the metaanalysis (Supplemental table 3). Latrine use increased across time in control sites $(\beta=0.83[0.48,1.18])$, and the increase over time was larger in the intervention sites than control sites $(\beta=0.43[0.16,0.70])$. Women tended to use latrines more than men $(\beta=0.51[0.47,0.54])$. Individuals in the schedule castes had the highest odds of latrine use. Those from Karnataka and Odisha had lower self-reported latrine use than those

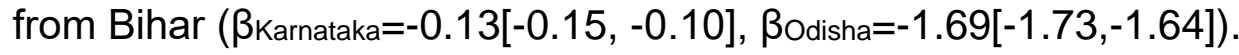

Results were also consistent when the imputed values were used for the Gujarat sample. Latrine use increased over time in control sites $(\beta=0.59[0.06,1.13])$, but there was a larger increase in intervention sites than control sites $(\beta=0.48[0.20,0.75])$. Women used latrines more than men $(\beta=0.36[0.07,0.66])$. And, individuals of the schedule castes had the highest reported latrine use. Individuals from Karnataka and Odisha had lower self-reported latrine use than those from Bihar ( $\beta_{\text {karnataka }}=-0.19[-0.29,-0.08\}$, $\beta$ odisha $=-1.52[-1.77,-1.25])$. However, those from Gujarat had similar self-reported latrine use to those from Bihar $(\beta=0.29[-0.19,0.77])$.

A sensitivity analysis was conducted for the meta-analysis in which $25 \%$ of those who self-reported latrine use was randomly assigned to be non-users to simulate misreporting. The results of the meta-analysis maintained statistical significance even if $25 \%$ of those who self-reported latrine use were not actually latrine users.

Table 4: Adjusted effect estimates of the intervention on selfreported latrine use

\begin{tabular}{lccc}
\hline & & P- & \\
Bihar $(\mathbf{n}=10364)$ & Coefficient & value & Cl \\
Period $^{1}$ & 0.985 & 0.000 & $0.567,1.401$ \\
Arm $^{2}$ & 0.049 & 0.906 & $-0.76,0.862$ \\
$\begin{array}{l}\text { Treatment by period } \\
\text { interaction }\end{array}$ & -0.249 & 0.308 & $-0.72,0.229$
\end{tabular}




\begin{tabular}{|c|c|c|c|}
\hline Female & 0.417 & 0.000 & $0.303,0.529$ \\
\hline Non-Hindu & 0.188 & 0.675 & $-0.69,1.066$ \\
\hline $\mathrm{Age}^{3}$ & -0.003 & 0.704 & $-0.01,0.010$ \\
\hline Age squared & 0.000 & 0.119 & $-0.00,3.312$ \\
\hline Household size $^{3}$ & -0.043 & 0.039 & $-0.08,-0.00$ \\
\hline \multicolumn{4}{|c|}{ Caste - Schedule Caste as referent } \\
\hline Scheduled tribe & -0.773 & 0.050 & $-1.54,-0.00$ \\
\hline General category & -1.180 & 0.007 & $-2.03,-0.32$ \\
\hline Other backward category & -0.720 & 0.046 & $-1.42,-0.01$ \\
\hline \multicolumn{4}{|l|}{ Karnataka $(n=19752)$} \\
\hline Period & 1.240 & 0.001 & $0.507,1.973$ \\
\hline Arm & 0.012 & 0.948 & $-0.33,0.359$ \\
\hline \multicolumn{4}{|l|}{ Treatment by period } \\
\hline interaction & 1.049 & 0.042 & $0.036,2.061$ \\
\hline Female & 0.544 & 0.000 & $0.430,0.656$ \\
\hline Non-Hindu & -0.012 & 0.954 & $-0.40,0.379$ \\
\hline Age & 0.029 & 0.000 & $0.018,0.039$ \\
\hline Age squared & 0.000 & 0.000 & $-0.00,-0.00$ \\
\hline Household size & -0.012 & 0.190 & $-0.03,0.005$ \\
\hline \multicolumn{4}{|c|}{ Caste - Schedule Caste as referent } \\
\hline Scheduled tribe & -0.538 & 0.002 & $-0.88,-0.19$ \\
\hline General category & -1.140 & 0.000 & $-1.51,-0.76$ \\
\hline Other backward category & -1.388 & 0.000 & $-1.73,-1.04$ \\
\hline Minority / other & -0.268 & 0.333 & $-0.81,0.274$ \\
\hline \multicolumn{4}{|l|}{ Odisha $(n=29271)$} \\
\hline Period & 0.654 & 0.000 & $0.510,0.797$ \\
\hline Arm & -0.053 & 0.748 & $-0.37,0.272$ \\
\hline \multicolumn{4}{|l|}{ Treatment by period } \\
\hline interaction & 0.423 & 0.000 & $0.189,0.655$ \\
\hline Female & 0.509 & 0.000 & $0.445,0.572$ \\
\hline Non-Hindu & -0.107 & 0.650 & $-0.56,0.353$ \\
\hline Age & -0.010 & 0.008 & $-0.01,-0.00$ \\
\hline Age squa & 0.000 & 0.558 & $-0.00,5.966$ \\
\hline Household size & -0.001 & 0.901 & $-0.01,0.016$ \\
\hline \multicolumn{4}{|c|}{ Caste - Schedule Caste as referent } \\
\hline General category & -1.150 & 0.005 & $-1.94,-0.35$ \\
\hline Other backward category & -1.140 & 0.000 & $-1.49,-0.78$ \\
\hline Minority / other & -0.565 & 0.000 & $-0.79,-0.33$ \\
\hline \multicolumn{4}{|l|}{ Gujarat $(n=16163)$} \\
\hline Period & -0.410 & 0.126 & $-0.93,0.115$ \\
\hline Arm & -0.296 & 0.347 & $-0.91,0.321$ \\
\hline \multicolumn{4}{|l|}{ Treatment by period } \\
\hline interactic & 0.904 & 0.023 & $0.122,1.685$ \\
\hline Female & 0.005 & 0.917 & $-0.08,0.097$ \\
\hline Non-Hindu & 1.758 & 0.095 & $-0.30,3.824$ \\
\hline
\end{tabular}




\begin{tabular}{|c|c|c|c|}
\hline Age & 0.015 & 0.006 & $0.004,0.025$ \\
\hline Age squared & 0.000 & 0.095 & $-0.00,1.983$ \\
\hline Household size & -0.019 & 0.491 & $-0.07,0.035$ \\
\hline \multicolumn{4}{|c|}{ Caste - Schedule Caste as referent } \\
\hline Scheduled tribe & -0.388 & 0.724 & $-2.54,1.770$ \\
\hline General category & -0.163 & 0.591 & $-0.75,0.429$ \\
\hline Other backward category & -0.532 & 0.052 & $-1.06,0.003$ \\
\hline Minority / other & -0.788 & 0.028 & $-1.48,-0.08$ \\
\hline \multicolumn{4}{|l|}{ Meta-analysis $(n=75550)$} \\
\hline Period & 0.660 & 0.003 & $0.225,1.093$ \\
\hline Arm & -0.048 & 0.101 & $-0.10,0.009$ \\
\hline \multicolumn{4}{|l|}{ Treatment by period } \\
\hline interaction & 0.509 & 0.000 & $0.257,0.761$ \\
\hline Female & 0.425 & 0.000 & $0.238,0.611$ \\
\hline Non-Hindu & 0.101 & 0.512 & $-0.20,0.404$ \\
\hline Age & 0.005 & 0.625 & $-0.01,0.027$ \\
\hline Age squared & 0.000 & 0.097 & $-0.00,2.556$ \\
\hline Household size & -0.011 & 0.110 & $-0.02,0.002$ \\
\hline \multicolumn{4}{|c|}{ Caste - Schedule Caste as referent } \\
\hline Scheduled tribe & -0.598 & 0.000 & $-0.84,-0.35$ \\
\hline General category & -1.067 & 0.000 & $-1.31,-0.81$ \\
\hline kward category & -1.238 & 0.000 & $-1.60,-0.87$ \\
\hline Minority / other & -0.669 & 0.000 & $-0.97,-0.36$ \\
\hline \multicolumn{4}{|l|}{ State - Bihar as referent } \\
\hline Karnataka & -0.151 & 0.000 & $-0.20,-0.10$ \\
\hline Odisha & -1.606 & 0.000 & $-1.77,-1.43$ \\
\hline Gujarat & 0.176 & 0.001 & $0.076,0.275$ \\
\hline
\end{tabular}

1. Coded as 0 for pre-intervention and 1 for post-intervention

2. Coded as 0 for control and 1 for treatment

3. Measure was standardized across the entire sample

Table 4: Adjusted effect estimates of the intervention on selfreported latrine use

\begin{tabular}{lccc}
\hline Bihar $(\mathbf{n = 1 0 3 6 4 )}$ & Coefficient & $\begin{array}{c}\text { P- } \\
\text { value }\end{array}$ & $\mathbf{C l}$ \\
Period $^{1}$ & 0.985 & 0.000 & $0.567,1.401$ \\
Arm $^{2}$ & 0.049 & 0.906 & $-0.76,0.862$ \\
Treatment by period & & & \\
interaction & -0.249 & 0.308 & $-0.72,0.229$ \\
Female & 0.417 & 0.000 & $0.303,0.529$ \\
Non-Hindu & 0.188 & 0.675 & $-0.69,1.066$ \\
Age $^{3}$ & -0.003 & 0.704 & $-0.01,0.010$ \\
$\quad$ Age squared & 0.000 & 0.119 & $-0.00,3.312$
\end{tabular}




\begin{tabular}{|c|c|c|c|}
\hline Household size $^{3}$ & -0.043 & 0.039 & $-0.08,-0.00$ \\
\hline \multicolumn{4}{|c|}{ Caste - Schedule Caste as referent } \\
\hline Scheduled tribe & -0.773 & 0.050 & $-1.54,-0.00$ \\
\hline General category & -1.180 & 0.007 & $-2.03,-0.32$ \\
\hline Other backward category & -0.720 & 0.046 & $-1.42,-0.01$ \\
\hline \multicolumn{4}{|l|}{ Karnataka $(n=19752)$} \\
\hline Period & 1.240 & 0.001 & $0.507,1.973$ \\
\hline Arm & 0.012 & 0.948 & $-0.33,0.359$ \\
\hline \multicolumn{4}{|l|}{ Treatment by period } \\
\hline interaction & 1.049 & 0.042 & $0.036,2.061$ \\
\hline Female & 0.544 & 0.000 & $0.430,0.656$ \\
\hline Non-Hindu & -0.012 & 0.954 & $-0.40,0.379$ \\
\hline Age & 0.029 & 0.000 & $0.018,0.039$ \\
\hline Age squared & 0.000 & 0.000 & $-0.00,-0.00$ \\
\hline Household size & -0.012 & 0.190 & $-0.03,0.005$ \\
\hline \multicolumn{4}{|c|}{ Caste - Schedule Caste as referent } \\
\hline Scheduled tribe & -0.538 & 0.002 & $-0.88,-0.19$ \\
\hline General category & -1.140 & 0.000 & $-1.51,-0.76$ \\
\hline Other backward category & -1.388 & 0.000 & $-1.73,-1.04$ \\
\hline Minority / other & -0.268 & 0.333 & $-0.81,0.274$ \\
\hline \multicolumn{4}{|l|}{ Odisha $(n=29271)$} \\
\hline Period & 0.654 & 0.000 & $0.510,0.797$ \\
\hline Arm & -0.053 & 0.748 & $-0.37,0.272$ \\
\hline \multicolumn{4}{|l|}{ Treatment by period } \\
\hline interaction & 0.423 & 0.000 & $0.189,0.655$ \\
\hline Female & 0.509 & 0.000 & $0.445,0.572$ \\
\hline Non-Hindu & -0.107 & 0.650 & $-0.56,0.353$ \\
\hline Age & -0.010 & 0.008 & $-0.01,-0.00$ \\
\hline Age squared & 0.000 & 0.558 & $-0.00,5.966$ \\
\hline Household size & -0.001 & 0.901 & $-0.01,0.016$ \\
\hline \multicolumn{4}{|c|}{ Caste - Schedule Caste as referent } \\
\hline General category & -1.150 & 0.005 & $-1.94,-0.35$ \\
\hline Other backward category & -1.140 & 0.000 & $-1.49,-0.78$ \\
\hline Minority / other & -0.565 & 0.000 & $-0.79,-0.33$ \\
\hline \multicolumn{4}{|l|}{ Gujarat (n=16163) } \\
\hline Period & -0.410 & 0.126 & $-0.93,0.115$ \\
\hline Arm & -0.296 & 0.347 & $-0.91,0.321$ \\
\hline \multicolumn{4}{|l|}{ Treatment by period } \\
\hline interaction & 0.904 & 0.023 & $0.122,1.685$ \\
\hline Female & 0.005 & 0.917 & $-0.08,0.097$ \\
\hline Non-Hindu & 1.758 & 0.095 & $-0.30,3.824$ \\
\hline Age & 0.015 & 0.006 & $0.004,0.025$ \\
\hline Age $\mathrm{s}$ & 0.000 & 0.095 & $-0.00,1.983$ \\
\hline Household size & -0.019 & 0.491 & $-0.07,0.035$ \\
\hline
\end{tabular}




\begin{tabular}{|c|c|c|c|}
\hline Scheduled tribe & -0.388 & 0.724 & $-2.54,1.770$ \\
\hline General category & -0.163 & 0.591 & $-0.75,0.429$ \\
\hline Other backward category & -0.532 & 0.052 & $-1.06,0.003$ \\
\hline Minority / other & -0.788 & 0.028 & $-1.48,-0.08$ \\
\hline \multicolumn{4}{|l|}{ Meta-analysis $(n=75550)$} \\
\hline Period & 0.660 & 0.003 & $0.225,1.093$ \\
\hline Arm & -0.048 & 0.101 & $-0.10,0.009$ \\
\hline \multicolumn{4}{|l|}{ Treatment by period } \\
\hline interaction & 0.509 & 0.000 & $0.257,0.761$ \\
\hline Female & 0.425 & 0.000 & $0.238,0.611$ \\
\hline Non-Hindu & 0.101 & 0.512 & $-0.20,0.404$ \\
\hline Age & 0.005 & 0.625 & $-0.01,0.027$ \\
\hline Age squared & 0.000 & 0.097 & $-0.00,2.556$ \\
\hline Household size & -0.011 & 0.110 & $-0.02,0.002$ \\
\hline \multicolumn{4}{|c|}{ Caste - Schedule Caste as referent } \\
\hline Scheduled tribe & -0.598 & 0.000 & $-0.84,-0.35$ \\
\hline General category & -1.067 & 0.000 & $-1.31,-0.81$ \\
\hline Other backward category & -1.238 & 0.000 & $-1.60,-0.87$ \\
\hline Minority / other & -0.669 & 0.000 & $-0.97,-0.36$ \\
\hline \multicolumn{4}{|l|}{ State - Bihar as referent } \\
\hline Karnataka & -0.151 & 0.000 & $-0.20,-0.10$ \\
\hline Odisha & -1.606 & 0.000 & $-1.77,-1.43$ \\
\hline Gujarat & 0.176 & 0.001 & $0.076,0.275$ \\
\hline
\end{tabular}


Figure 1: Effects of the four interventions on self-reported latrine use. Presented as odds ratios as calculated by the difference-in-difference.

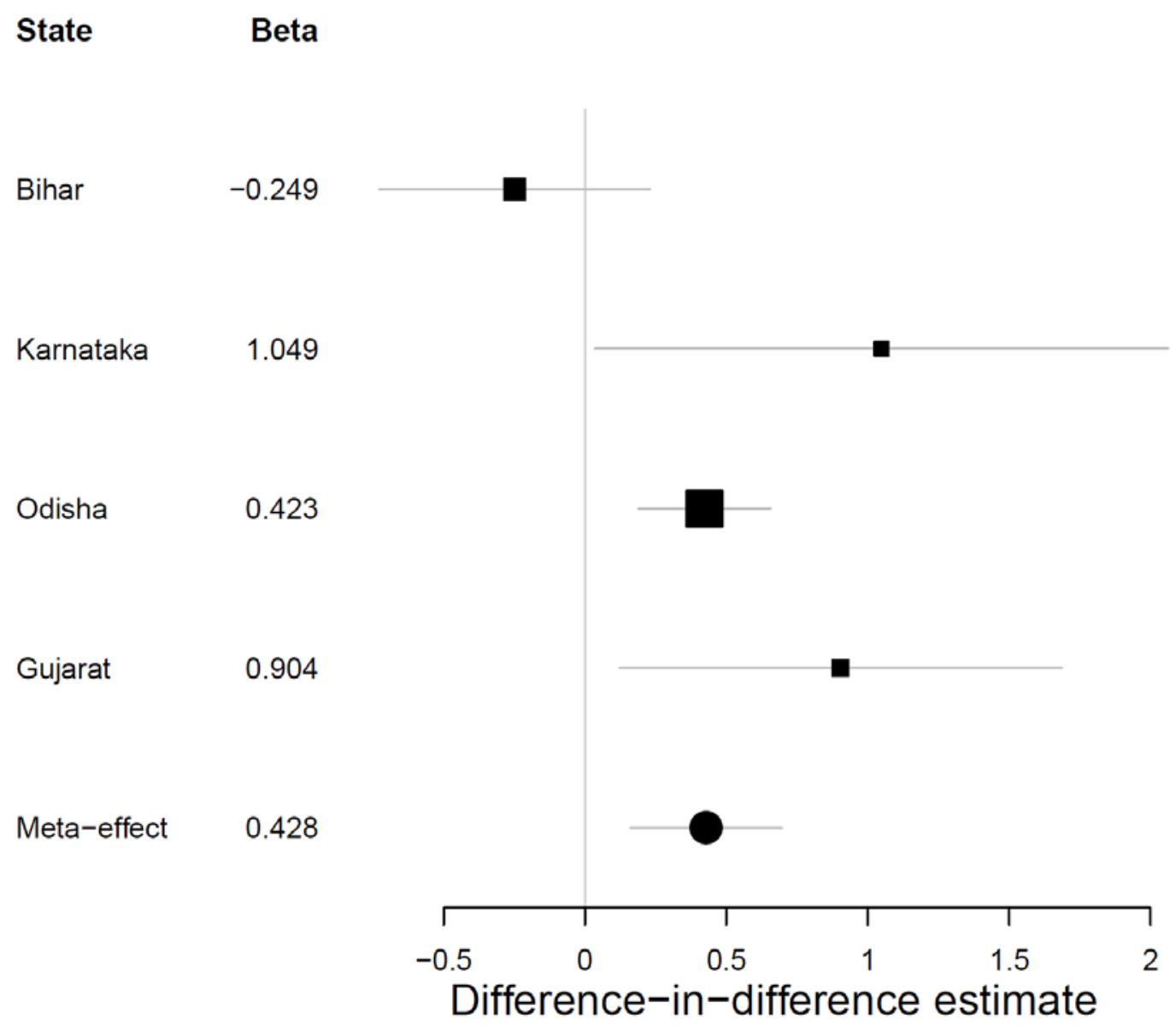

Note: Squares represent estimates for individual studies and circles are for summary estimates. The size is inversely proportional to the width of the confidence interval. 


\subsection{Latrine observation index}

\subsubsection{Univariate analysis}

Latrine observation index scores increased in control villages over the course of the intervention in Bihar $(\beta=0.24[0.16,0.32])$. However, they decreased in Odisha and Gujarat ( $\beta$ odisha $\left.=-0.14[-0.21,-0.8], \beta_{\text {Gujarat }}=-1.11[-1.30,-0.92]\right)$. There was no change in Karnataka or the average effect $\left(\beta_{\text {Karnataka }}=0.01[-0.15,0.17], \beta_{\text {Average effect }}=-0.7[-\right.$ $0.24,0.11])$. There was no difference in the change in the latrine observation index scores between intervention and control sites $\left(\beta_{\text {Bihar }}=-0.5[-0.18,0.08], \beta_{\text {Karnataka }}=0.09[-\right.$ $0.17,0.34], \beta_{\text {odisha }}=-0.05[-0.15,0.05], \beta_{\text {Gujarat }}=-0.03[-0.31,0.26], \beta_{\text {Average effect }}=0.04[-$ $0.05,0.13])$.

Table 5: Unadjusted effect estimates of the intervention on the latrine observation index scores

\begin{tabular}{|c|c|c|c|}
\hline Bihar $(n=10498)$ & Coefficient & $\begin{array}{c}\text { P- } \\
\text { value }\end{array}$ & $\begin{array}{c}\mathbf{C l} \\
01590\end{array}$ \\
\hline Period $^{1}$ & 0.238 & 0.000 & $\begin{array}{l}0.1590 \\
0.3176 \\
-0.247\end{array}$ \\
\hline $\operatorname{Arm}^{2}$ & -0.050 & 0.621 & $\begin{array}{l}0.1479 \\
-0.181\end{array}$ \\
\hline $\begin{array}{l}\text { Treatment by period interaction } \\
\text { Karnataka ( } \mathrm{n=19547)}\end{array}$ & -0.049 & 0.472 & 0.0838 \\
\hline (a) & 0.012 & 0.883 & $\begin{array}{l}-0.149 \\
0.1735 \\
-0.225\end{array}$ \\
\hline Arm & -0.017 & 0.874 & $\begin{array}{l}0.1915 \\
-0.166\end{array}$ \\
\hline $\begin{array}{l}\text { Treatment by period interaction } \\
\text { Odisha }(\mathrm{n}=\mathbf{2 8 3 2 7})\end{array}$ & 0.088 & 0.497 & 0.3436 \\
\hline Period & -0.145 & 0.000 & $\begin{array}{l}-0.212,-0.077 \\
-0.102\end{array}$ \\
\hline Arm & 0.030 & 0.661 & $\begin{array}{l}0.1624 \\
-0.154\end{array}$ \\
\hline $\begin{array}{l}\text { Treatment by period interaction } \\
\text { Gujarat }(n=16202)\end{array}$ & -0.052 & 0.327 & 0.0515 \\
\hline Period & -1.109 & 0.000 & $\begin{array}{c}-1.302,-0.915 \\
-0.134\end{array}$ \\
\hline Arm & 0.119 & 0.357 & $\begin{array}{l}0.3728 \\
-0.309\end{array}$ \\
\hline $\begin{array}{l}\text { Treatment by period interaction } \\
\text { Meta-analysis }(n=74574)\end{array}$ & -0.026 & 0.856 & 0.2572 \\
\hline Period & -0.067 & 0.457 & $\begin{array}{l}-0.242 \\
0.1092\end{array}$ \\
\hline
\end{tabular}




\begin{tabular}{llll} 
Arm & & & -0.047, \\
& -0.004 & 0.871 & 0.0404 \\
Treatment by period interaction & 0.040 & 0.380 & -0.048, \\
\hline
\end{tabular}

1. Coded as 0 for pre-intervention and 1 for post-intervention

2. Coded as 0 for control and 1 for treatment

Table 5: Unadjusted effect estimates of the intervention on the latrine observation index scores

\begin{tabular}{|c|c|c|c|}
\hline Bihar $(n=10498)$ & Coefficient & P-value & $\mathrm{Cl}$ \\
\hline Period $^{1}$ & 0.238 & 0.000 & $0.1590,0.3176$ \\
\hline $\mathrm{Arm}^{2}$ & -0.050 & 0.621 & $-0.247,0.1479$ \\
\hline $\begin{array}{l}\text { Treatment by period } \\
\text { interaction } \\
\text { Karnataka }(n=19547)\end{array}$ & -0.049 & 0.472 & $-0.181,0.0838$ \\
\hline Period & 0.012 & 0.883 & $-0.149,0.1735$ \\
\hline Arm & -0.017 & 0.874 & $-0.225,0.1915$ \\
\hline $\begin{array}{l}\text { Treatment by period } \\
\text { interaction }\end{array}$ & 0.088 & 0.497 & $-0.166,0.3436$ \\
\hline $\begin{array}{l}\text { Odisha }(n=28327) \\
\text { Period }\end{array}$ & -0.145 & 0.000 & $-0.212,-0.077$ \\
\hline Arm & 0.030 & 0.661 & $-0.102,0.1624$ \\
\hline $\begin{array}{l}\text { Treatment by period } \\
\text { interaction } \\
\text { Gujarat }(n=16202)\end{array}$ & -0.052 & 0.327 & $-0.154,0.0515$ \\
\hline Period & -1.109 & 0.000 & $-1.302,-0.915$ \\
\hline $\begin{array}{l}\text { Arm } \\
\text { Treatment by period }\end{array}$ & 0.119 & 0.357 & $-0.134,0.3728$ \\
\hline $\begin{array}{l}\text { interaction } \\
\text { Meta-analysis }(n=74574)\end{array}$ & -0.026 & 0.856 & $-0.309,0.2572$ \\
\hline Period & -0.067 & 0.457 & $-0.242,0.1092$ \\
\hline Arm & -0.004 & 0.871 & $-0.047,0.0404$ \\
\hline $\begin{array}{l}\text { Treatment by period } \\
\text { interaction }\end{array}$ & 0.040 & 0.380 & $-0.048,0.1284$ \\
\hline
\end{tabular}

1. Coded as 0 for pre-intervention and 1 for post-intervention

2. Coded as 0 for control and 1 for treatment

\subsubsection{Multivariate analysis}

The latrine observation index scores increased in control villages in Bihar over the intervention period (Table $6, \beta=0.25[0.16,0.33]$ ); however, the index scores decreased in Odisha and Gujarat ( $\beta$ odisha $=-0.14[-0.20,-0.07]$, $\beta$ Gujarat $=-1.10[-1.29,-0.90])$. There was 
no change in Karnataka or the average effect estimate ( $\beta_{\text {Karnataka }}=0.01[-0.14,0.18]$, $\left.\beta_{\text {Average effect }}=-0.20[-0.58,0.20]\right)$. There were no significant differences in the change in index scores between intervention and control arms in any of the studies ( $\beta_{\text {Bihar }}=-0.6[-$ $0.19,0.7], \beta_{\text {Karnataka }}=0.08[-0.17,0.34]$, $\beta_{\text {odisha }}=-0.05[-0.15,0.5], \beta_{\text {Gujarat }}=-0.03[-0.31,0.25]$, $\left.\beta_{\text {meta }}=0.02[-0.06,0.09]\right)$.

In Karnataka and Odisha, older individuals tended to inhabit households with lower latrine observation index scores $\left(\beta_{\text {Karnataka }}=0.01[0.00,0.01]\right.$, $\beta$ odisha $\left.=0.01[0.00,0.01]\right)$, but this was not seen in Bihar, Gujarat, or the average effect estimate ( $\beta_{\text {Bihar }}=-0.00[-$ $0.00,0.00], \beta_{\text {Gujarat }}=0.00[-0.00,0.00]$, $\left.\beta_{\text {Average effect }}=0.00[-0.00,0.01]\right)$. Individuals of scheduled castes tended to inhabit households with the highest latrine observation index scores. Households with higher latrine observation index scores tended to be in Karnataka and Gujarat ( $\beta$ Karnataka=0.98[0.95,1.01], $\beta$ Gujarat1.11[1.01,1.20]) rather than Bihar. Households with lower latrine observation index scores tended to be in Odisha rather than Bihar ( $\beta=-0.32[-0.39,-0.25])$.

\subsubsection{Multivariate sensitivity analysis}

These results remained the same under alternate sample specifications for the Gujarat data (Supplemental table 4, Supplemental figure 2). When the sample was restricted to villages sampled both before and after the intervention, latrine observation index scores decreased over the course of the intervention in control sites ( $\beta=-1.11[-1.29,-0.91])$, with no significant difference in the decrease between intervention and control sites ( $\beta=-$ $0.01[-0.09,0.08])$. Similarly, when pre-intervention values were imputed for individuals sampled after the intervention, latrine observation index scores were found to decrease over time in control sites $(\beta=-1.15[-1.22,-1.06])$, with no significant difference in the decrease between intervention and control sites $(\beta=-0.01[-0.07,0.06])$.

Alternate sample specifications did not affect the primary findings of the meta-analysis (Supplemental table 5). When Gujarat was removed from the analysis there were no significant changes in latrine observation index scores over time in control sites $(\beta=-$ $0.02[-0.19,0.16])$ or differences in the change between intervention and control villages $(\beta=-0.01[-0.09,0.03])$. Households with higher latrine observation index scores tended to be in Karnataka rather than Bihar $(\beta=0.99[0.98,1.02])$, and households with lower scores tended to be in Odisha rather than Bihar $(\beta=-0.36[-0.39,-0.32])$. Similarly, when imputed values were used for the estimation of the pre-intervention values of individuals sampled in Gujarat after the intervention, there were no significant changes in latrine observation index scores over time in control sites $(\beta=-0.36[-0.98,0.26])$ or differences in the change between intervention and control villages $(\beta=-0.01[-0.01,0.07])$. Households with higher latrine observation index scores tended to be in Karnataka or Gujarat rather than Bihar ( $\beta_{\text {Karnataka }}=0.98[0.96,1.01]$, $\beta$ Gujarat $\left.=1.41[1.35,1.47]\right)$. Households with lower scores tended to be in Odisha rather than Bihar $(\beta=-0.31[-0.40 .-021])$. 
Table 4: Adjusted effect estimates of the intervention on selfreported latrine use

\begin{tabular}{lccc}
\hline Bihar (n=10364) & Coefficient & $\begin{array}{c}\text { P- } \\
\text { value }\end{array}$ & $\mathbf{C l}$ \\
Period $^{1}$ & 0.985 & 0.000 & $0.567,1.401$ \\
Arm $^{2}$ & 0.049 & 0.906 & $-0.76,0.862$ \\
Treatment by period & & & \\
interaction & -0.249 & 0.308 & $-0.72,0.229$ \\
Female & 0.417 & 0.000 & $0.303,0.529$ \\
Non-Hindu & 0.188 & 0.675 & $-0.69,1.066$ \\
Age $^{3}$ & -0.003 & 0.704 & $-0.01,0.010$ \\
$\quad$ Age squared & 0.000 & 0.119 & $-0.00,3.312$ \\
Household size & -0.043 & 0.039 & $-0.08,-0.00$ \\
Caste - Schedule Caste as referent & & & \\
$\quad$ Scheduled tribe & -0.773 & 0.050 & $-1.54,-0.00$ \\
$\quad$ General category & -1.180 & 0.007 & $-2.03,-0.32$ \\
$\quad$ Other backward category & -0.720 & 0.046 & $-1.42,-0.01$ \\
Karnataka (n=19752) & & & \\
Period & 1.240 & 0.001 & $0.507,1.973$ \\
Arm & 0.012 & 0.948 & $-0.33,0.359$ \\
Treatment by period & & & \\
interaction & 1.049 & 0.042 & $0.036,2.061$ \\
Female & 0.544 & 0.000 & $0.430,0.656$ \\
Non-Hindu & -0.012 & 0.954 & $-0.40,0.379$ \\
Age & 0.029 & 0.000 & $0.018,0.039$ \\
$\quad$ Age squared & 0.000 & 0.000 & $-0.00,-0.00$ \\
Household size & -0.012 & 0.190 & $-0.03,0.005$ \\
Caste - Schedule Caste as referent & & & \\
$\quad$ Scheduled tribe & -0.538 & 0.002 & $-0.88,-0.19$ \\
$\quad$ General category & -1.140 & 0.000 & $-1.51,-0.76$ \\
$\quad$ Other backward category & -1.388 & 0.000 & $-1.73,-1.04$ \\
$\quad$ Minority / other & -0.268 & 0.333 & $-0.81,0.274$ \\
Odisha (n=29271) & & & \\
Period & 0.654 & 0.000 & $0.510,0.797$ \\
Arm & -0.053 & 0.748 & $-0.37,0.272$ \\
Treatment by period & & & \\
interaction & -1.150 & 0.005 & $-1.94,-0.35$ \\
Female & -1.140 & 0.000 & $-1.49,-0.78$ \\
Non-Hindu & & & \\
Age & 0.423 & 0.000 & $0.189,0.655$ \\
$\quad$ Age squared & 0.509 & 0.000 & $0.445,0.572$ \\
Household size & -0.107 & 0.650 & $-0.56,0.353$ \\
Caste - Schedule Caste as referent & -0.010 & 0.008 & $-0.01,-0.00$ \\
$\quad$ General category & 0.000 & 0.558 & $-0.00,5.966$ \\
Other backward category & & 0.901 & $-0.01,0.016$ \\
& & &
\end{tabular}




\begin{tabular}{|c|c|c|c|}
\hline Minority / other & -0.565 & 0.000 & $-0.79,-0.33$ \\
\hline \multicolumn{4}{|l|}{ Gujarat (n=16163) } \\
\hline Period & -0.410 & 0.126 & $-0.93,0.115$ \\
\hline Arm & -0.296 & 0.347 & $-0.91,0.321$ \\
\hline \multicolumn{4}{|l|}{ Treatment by period } \\
\hline interaction & 0.904 & 0.023 & $0.122,1.685$ \\
\hline Female & 0.005 & 0.917 & $-0.08,0.097$ \\
\hline Non-Hindu & 1.758 & 0.095 & $-0.30,3.824$ \\
\hline Age & 0.015 & 0.006 & $0.004,0.025$ \\
\hline Age squared & 0.000 & 0.095 & $-0.00,1.983$ \\
\hline Household size & -0.019 & 0.491 & $-0.07,0.035$ \\
\hline \multicolumn{4}{|c|}{ Caste - Schedule Caste as referent } \\
\hline Scheduled tribe & -0.388 & 0.724 & $-2.54,1.770$ \\
\hline General category & -0.163 & 0.591 & $-0.75,0.429$ \\
\hline Other backward category & -0.532 & 0.052 & $-1.06,0.003$ \\
\hline Minority / other & -0.788 & 0.028 & $-1.48,-0.08$ \\
\hline \multicolumn{4}{|l|}{ Meta-analysis $(n=75550)$} \\
\hline Period & 0.660 & 0.003 & $0.225,1.093$ \\
\hline Arm & -0.048 & 0.101 & $-0.10,0.009$ \\
\hline \multicolumn{4}{|l|}{ Treatment by period } \\
\hline $\begin{array}{l}\text { interaction } \\
\text { Female }\end{array}$ & 0.425 & 0.000 & $0.238,0.611$ \\
\hline Non-Hindu & 0.101 & 0.512 & $-0.20,0.404$ \\
\hline Age & 0.005 & 0.625 & $-0.01,0.027$ \\
\hline Age squared & 0.000 & 0.097 & $-0.00,2.556$ \\
\hline Household size & -0.011 & 0.110 & $-0.02,0.002$ \\
\hline \multicolumn{4}{|c|}{ Caste - Schedule Caste as referent } \\
\hline Scheduled tribe & -0.598 & 0.000 & $-0.84,-0.35$ \\
\hline General category & -1.067 & 0.000 & $-1.31,-0.81$ \\
\hline Other backward category & -1.238 & 0.000 & $-1.60,-0.87$ \\
\hline Minority / other & -0.669 & 0.000 & $-0.97,-0.36$ \\
\hline \multicolumn{4}{|l|}{ State - Bihar as referent } \\
\hline Karnataka & -0.151 & 0.000 & $-0.20,-0.10$ \\
\hline Odisha & -1.606 & 0.000 & $-1.77,-1.43$ \\
\hline Gujarat & 0.176 & 0.001 & $0.076,0.275$ \\
\hline
\end{tabular}

1. Coded as 0 for pre-intervention and 1 for post-intervention

2. Coded as 0 for control and 1 for treatment

3. Measure was standardized across the entire sample 
Table 6: Adjusted effect estimates of the intervention on the latrine observation index scores

\begin{tabular}{|c|c|c|c|}
\hline Bihar $(n=10462)$ & Coefficient & P-value & $\mathrm{Cl}$ \\
\hline Period $^{i}$ & 0.245 & 0.000 & $0.163,0.325$ \\
\hline $\mathrm{Arm}^{2}$ & -0.034 & 0.734 & $-0.23,0.164$ \\
\hline \multicolumn{4}{|l|}{ Treatment by period } \\
\hline interaction & -0.059 & 0.374 & $-0.19,0.071$ \\
\hline Female & 0.008 & 0.409 & $-0.01,0.028$ \\
\hline Non-Hindu & 0.040 & 0.679 & $-0.15,0.232$ \\
\hline $\mathrm{Age}^{3}$ & -0.001 & 0.415 & $-0.00,0.001$ \\
\hline Age squared & 0.000 & 0.899 & $-3.49,3.977$ \\
\hline Household size ${ }^{3}$ & -0.010 & 0.092 & $-0.02,0.001$ \\
\hline \multicolumn{4}{|c|}{ Caste - Schedule Caste as referent } \\
\hline Scheduled tribe & -0.252 & 0.003 & $-0.41,-0.08$ \\
\hline General category & -0.433 & 0.000 & $-0.62,-0.24$ \\
\hline Other backward category & -0.173 & 0.265 & $-0.47,0.131$ \\
\hline \multicolumn{4}{|l|}{ Karnataka $(n=19418)$} \\
\hline Period & 0.014 & 0.865 & $-0.14,0.177$ \\
\hline Arm & -0.004 & 0.971 & $-0.20,0.198$ \\
\hline \multicolumn{4}{|l|}{ Treatment by period } \\
\hline interaction & 0.085 & 0.517 & $-0.17,0.340$ \\
\hline Female & -0.008 & 0.482 & $-0.02,0.013$ \\
\hline Non-Hindu & -0.064 & 0.417 & $-0.21,0.090$ \\
\hline Age & 0.006 & 0.009 & $0.001,0.010$ \\
\hline Age squared & 0.000 & 0.009 & $-0.00,-2.05$ \\
\hline Household size & -0.002 & 0.685 & $-0.01,0.007$ \\
\hline \multicolumn{4}{|c|}{ Caste - Schedule Caste as referent } \\
\hline Scheduled tribe & -0.094 & 0.024 & $-0.17,-0.01$ \\
\hline General category & -0.285 & 0.000 & $-0.39,-0.17$ \\
\hline Other backward category & -0.376 & 0.000 & $-0.50,-0.24$ \\
\hline Minority / other & 0.012 & 0.923 & $-0.23,0.256$ \\
\hline \multicolumn{4}{|l|}{ Odisha $(n=27213)$} \\
\hline Period & -0.139 & 0.000 & $-0.20,-0.07$ \\
\hline Arm & 0.024 & 0.707 & $-0.10,0.151$ \\
\hline \multicolumn{4}{|l|}{ Treatment by period } \\
\hline interaction & -0.054 & 0.301 & $-0.15,0.048$ \\
\hline Female & -0.006 & 0.439 & $-0.02,0.009$ \\
\hline Non-Hindu & -0.131 & 0.060 & $-0.26,0.005$ \\
\hline Age & 0.005 & 0.000 & $0.003,0.007$ \\
\hline Age squared & 0.000 & 0.009 & $-6.76,-9.46$ \\
\hline Household size & 0.006 & 0.134 & $-0.00,0.013$ \\
\hline \multicolumn{4}{|c|}{ Caste - Schedule Caste as referent } \\
\hline General category & -0.453 & 0.002 & $-0.73,-0.16$ \\
\hline
\end{tabular}




\begin{tabular}{|c|c|c|c|}
\hline Other backward category & -0.317 & 0.000 & $-0.42,-0.20$ \\
\hline Minority / other & -0.366 & 0.000 & $-0.47,-0.25$ \\
\hline \multicolumn{4}{|l|}{ Gujarat $(n=16202)$} \\
\hline Period & -1.097 & 0.000 & $-1.28,-0.90$ \\
\hline Arm & 0.119 & 0.359 & $-0.13,0.373$ \\
\hline \multicolumn{4}{|l|}{ Treatment by period } \\
\hline interaction & -0.030 & 0.838 & $-0.31,0.254$ \\
\hline Female & 0.006 & 0.420 & $-0.00,0.022$ \\
\hline Non-Hindu & 0.115 & 0.507 & $-0.22,0.453$ \\
\hline Age & 0.000 & 0.749 & $-0.00,0.001$ \\
\hline Age squared & 0.000 & 0.170 & $-6.89,3.914$ \\
\hline Household size & -0.005 & 0.528 & $-0.02,0.010$ \\
\hline \multicolumn{4}{|c|}{ Caste - Schedule Caste as referent } \\
\hline Scheduled tribe & -0.060 & 0.788 & $-0.49,0.378$ \\
\hline General category & 0.031 & 0.722 & $-0.14,0.203$ \\
\hline Other backward category & -0.011 & 0.891 & $-0.17,0.148$ \\
\hline Minority / other & -0.192 & 0.040 & $-0.37,-0.00$ \\
\hline \multicolumn{4}{|l|}{ Meta-analysis $(n=7329)$} \\
\hline Period & -0.195 & 0.328 & $-0.58,0.195$ \\
\hline Arm & 0.005 & 0.722 & $-0.02,0.034$ \\
\hline \multicolumn{4}{|l|}{ Treatment by period } \\
\hline interaction & 0.016 & 0.696 & $-0.06,0.094$ \\
\hline Female & -0.001 & 0.906 & $-0.01,0.009$ \\
\hline Non-Hindu & 0.036 & 0.555 & $-0.08,0.157$ \\
\hline Age & 0.002 & 0.187 & $-0.00,0.005$ \\
\hline Age squared & 0.000 & 0.364 & $-4.98,1.829$ \\
\hline Household size & -0.001 & 0.735 & $-0.00,0.005$ \\
\hline \multicolumn{4}{|c|}{ Caste - Schedule Caste as referent } \\
\hline Scheduled tribe & -0.138 & 0.000 & $-0.21,-0.06$ \\
\hline General category & -0.318 & 0.000 & $-0.37,-0.25$ \\
\hline Other backward category & -0.301 & 0.001 & $-0.47,-0.13$ \\
\hline Minority / other & -0.369 & 0.000 & $-0.52,-0.20$ \\
\hline \multicolumn{4}{|l|}{ State - Bihar as referent } \\
\hline Karnataka & 0.981 & 0.000 & $0.952,1.009$ \\
\hline Odisha & -0.324 & 0.000 & $-0.39,-0.25$ \\
\hline Gujarat & 1.105 & 0.000 & $1.009,1.200$ \\
\hline \multicolumn{4}{|c|}{ 1. Coded as 0 for pre-intervention and 1 for post-intervention } \\
\hline ( & & & \\
\hline
\end{tabular}


Table 6: Adjusted effect estimates of the intervention on the latrine observation index scores

\begin{tabular}{|c|c|c|c|}
\hline Bihar $(n=10462)$ & Coefficient & P-value & $\mathrm{Cl}$ \\
\hline Period $^{i}$ & 0.245 & 0.000 & $0.163,0.325$ \\
\hline $\mathrm{Arm}^{2}$ & -0.034 & 0.734 & $-0.23,0.164$ \\
\hline \multicolumn{4}{|l|}{ Treatment by period } \\
\hline interaction & -0.059 & 0.374 & $-0.19,0.071$ \\
\hline Female & 0.008 & 0.409 & $-0.01,0.028$ \\
\hline Non-Hindu & 0.040 & 0.679 & $-0.15,0.232$ \\
\hline $\mathrm{Age}^{3}$ & -0.001 & 0.415 & $-0.00,0.001$ \\
\hline Age squared & 0.000 & 0.899 & $-3.49,3.977$ \\
\hline Household size ${ }^{3}$ & -0.010 & 0.092 & $-0.02,0.001$ \\
\hline \multicolumn{4}{|c|}{ Caste - Schedule Caste as referent } \\
\hline Scheduled tribe & -0.252 & 0.003 & $-0.41,-0.08$ \\
\hline General category & -0.433 & 0.000 & $-0.62,-0.24$ \\
\hline Other backward category & -0.173 & 0.265 & $-0.47,0.131$ \\
\hline \multicolumn{4}{|l|}{ Karnataka $(n=19418)$} \\
\hline Period & 0.014 & 0.865 & $-0.14,0.177$ \\
\hline Arm & -0.004 & 0.971 & $-0.20,0.198$ \\
\hline \multicolumn{4}{|l|}{ Treatment by period } \\
\hline interaction & 0.085 & 0.517 & $-0.17,0.340$ \\
\hline Female & -0.008 & 0.482 & $-0.02,0.013$ \\
\hline Non-Hindu & -0.064 & 0.417 & $-0.21,0.090$ \\
\hline Age & 0.006 & 0.009 & $0.001,0.010$ \\
\hline Age squared & 0.000 & 0.009 & $-0.00,-2.05$ \\
\hline Household size & -0.002 & 0.685 & $-0.01,0.007$ \\
\hline \multicolumn{4}{|c|}{ Caste - Schedule Caste as referent } \\
\hline Scheduled tribe & -0.094 & 0.024 & $-0.17,-0.01$ \\
\hline General category & -0.285 & 0.000 & $-0.39,-0.17$ \\
\hline Other backward category & -0.376 & 0.000 & $-0.50,-0.24$ \\
\hline Minority / other & 0.012 & 0.923 & $-0.23,0.256$ \\
\hline \multicolumn{4}{|l|}{ Odisha $(n=27213)$} \\
\hline Period & -0.139 & 0.000 & $-0.20,-0.07$ \\
\hline Arm & 0.024 & 0.707 & $-0.10,0.151$ \\
\hline \multicolumn{4}{|l|}{ Treatment by period } \\
\hline interaction & -0.054 & 0.301 & $-0.15,0.048$ \\
\hline Female & -0.006 & 0.439 & $-0.02,0.009$ \\
\hline Non-Hindu & -0.131 & 0.060 & $-0.26,0.005$ \\
\hline Age & 0.005 & 0.000 & $0.003,0.007$ \\
\hline Age squared & 0.000 & 0.009 & $-6.76,-9.46$ \\
\hline Household size & 0.006 & 0.134 & $-0.00,0.013$ \\
\hline \multicolumn{4}{|c|}{ Caste - Schedule Caste as referent } \\
\hline General category & -0.453 & 0.002 & $-0.73,-0.16$ \\
\hline
\end{tabular}




\begin{tabular}{|c|c|c|c|}
\hline Other backward category & -0.317 & 0.000 & $-0.42,-0.20$ \\
\hline Minority / other & -0.366 & 0.000 & $-0.47,-0.25$ \\
\hline \multicolumn{4}{|l|}{ Gujarat $(n=16202)$} \\
\hline Period & -1.097 & 0.000 & $-1.28,-0.90$ \\
\hline Arm & 0.119 & 0.359 & $-0.13,0.373$ \\
\hline \multicolumn{4}{|l|}{ Treatment by period } \\
\hline interaction & -0.030 & 0.838 & $-0.31,0.254$ \\
\hline Female & 0.006 & 0.420 & $-0.00,0.022$ \\
\hline Non-Hindu & 0.115 & 0.507 & $-0.22,0.453$ \\
\hline Age & 0.000 & 0.749 & $-0.00,0.001$ \\
\hline Age squared & 0.000 & 0.170 & $-6.89,3.914$ \\
\hline Household size & -0.005 & 0.528 & $-0.02,0.010$ \\
\hline \multicolumn{4}{|c|}{ Caste - Schedule Caste as referent } \\
\hline Scheduled tribe & -0.060 & 0.788 & $-0.49,0.378$ \\
\hline General category & 0.031 & 0.722 & $-0.14,0.203$ \\
\hline Other backward category & -0.011 & 0.891 & $-0.17,0.148$ \\
\hline Minority / other & -0.192 & 0.040 & $-0.37,-0.00$ \\
\hline \multicolumn{4}{|l|}{ Meta-analysis $(n=7329)$} \\
\hline Period & -0.195 & 0.328 & $-0.58,0.195$ \\
\hline Arm & 0.005 & 0.722 & $-0.02,0.034$ \\
\hline \multicolumn{4}{|l|}{ Treatment by period } \\
\hline interaction & 0.016 & 0.696 & $-0.06,0.094$ \\
\hline Female & -0.001 & 0.906 & $-0.01,0.009$ \\
\hline Non-Hindu & 0.036 & 0.555 & $-0.08,0.157$ \\
\hline Age & 0.002 & 0.187 & $-0.00,0.005$ \\
\hline Age squared & 0.000 & 0.364 & $-4.98,1.829$ \\
\hline Household size & -0.001 & 0.735 & $-0.00,0.005$ \\
\hline \multicolumn{4}{|c|}{ Caste - Schedule Caste as referent } \\
\hline Scheduled tribe & -0.138 & 0.000 & $-0.21,-0.06$ \\
\hline General category & -0.318 & 0.000 & $-0.37,-0.25$ \\
\hline Other backward category & -0.301 & 0.001 & $-0.47,-0.13$ \\
\hline Minority / other & -0.369 & 0.000 & $-0.52,-0.20$ \\
\hline \multicolumn{4}{|l|}{ State - Bihar as referent } \\
\hline Karnataka & 0.981 & 0.000 & $0.952,1.009$ \\
\hline Odisha & -0.324 & 0.000 & $-0.39,-0.25$ \\
\hline Gujarat & 1.105 & 0.000 & $1.009,1.200$ \\
\hline \multicolumn{4}{|c|}{ 1. Coded as 0 for pre-intervention and 1 for post-intervention } \\
\hline ( & & & \\
\hline
\end{tabular}


Figure 2: Effects of the four interventions on observed latrine use. Presented as odds ratios as calculated by the difference-in-difference.

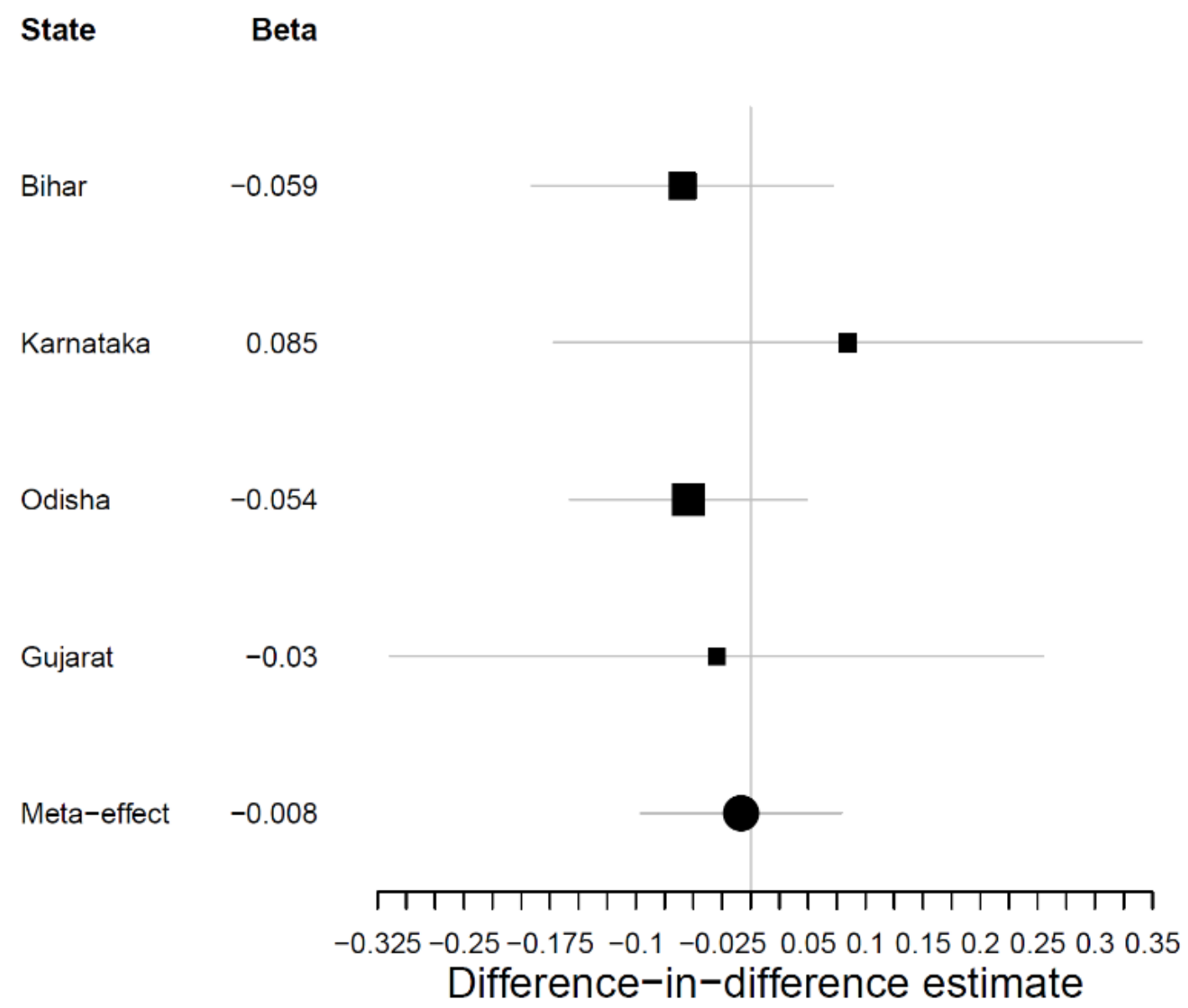

Squares represent estimates for individual studies and circles are for summary estimates. The size is inversely proportional to the width of the confidence interval. 


\section{Discussion}

Through a difference-in-difference meta-analysis using the individual-participant data, we found that the four behavioural-science informed interventions, when considered collectively, had a positive average effect on self-reported latrine use ( $\beta_{\text {Average }}$ effect $=0.51[0.26,0.76])$. However, there was no collective effect on use when using the household-level latrine observation index. The Swachh Bharat Mission is likely responsible for the increase in self-reported latrine use in control areas, but the increase in the intervention arms that was beyond that observed in the control arms can be attributed to the evaluated interventions. This finding is consistent with the results the individual studies reported in their independent analyses, which indicated positive effects on latrine use in Karnataka, Odisha, and Gujarat, with null effects in Bihar (Caruso et al., 2019b; Chuahan et al., 2019; Friedrich et al., 2019; International Initiative for Impact Evaluation, 2020; Schmidt, Wolf-peter, Chauhan et al., 2020; Viswanathan et al., 2019). However, the calculation of the average effect across all four studies has the added benefit of being both statistically more powerful and applicable to a broader set of contexts. This average effect may more closely reflect the impact that policy makers could expect should they attempt to introduce tailored and piloted behaviour-science informed interventions in their own, local context.

This study builds on the work of the four independent impact (Caruso et al., 2019b; Chuahan et al., 2019; Friedrich et al., 2019; International Initiative for Impact Evaluation, 2020; Schmidt, Wolf-peter, Chauhan et al., 2020; Viswanathan et al., 2019). By using a common analytical approach to synthesize findings across studies, we are able to identify similarities in predictors of latrine use, such as sex and caste, and differences in predictors of latrine use, such as age, across these four states. We are also able to directly compare the treatment effects of the four different interventions. Our use of a novel methodology (prospective, individual-participant meta-analysis) allows us to quantify an average treatment effect. These analyses would not have been possible if the studies had not been designed from the beginning to allow for comparison by using the same measures. This co-creation of four unique research projects was a considerable effort for each research team, but ultimately resulted in a rich body of data that allows for deeper cross-context conclusions than would have been possible had each study been conducted fully independently. Future programs that would like to understand cross-context effects of interventions can adopt a similar, collaborative approach.

By analyzing each of the four studies using a common method, we are able to identify heterogeneity in results that would not have been seen otherwise because the results of each impact evaluation have been presented separately using different methods. The average treatment effect shows that, across studies, age has no effect on latrine use. However, by taking the same analytical approach to quantifying impact across the four studies, we find that age, in fact, has a different relationship with latrine use in the four 
settings. In Odisha, older individuals are less likely to use latrines, consistent with previous work (Sinha et al., 2017). In contrast, in Karnataka and Gujarat, older individuals are more likely to use latrines. The variation in our results reflect the immense diversity of India in terms of socioeconomic traits and culture. This variation also indicates the benefit of calculating individual study effects in addition to average effects. However, even this may not capture the true variation in open defecation behaviour, which is related to a complex interaction between age, sex, and social status (Routray et al., 2015).

Consistent with other work, we find that women self-report latrine use more than men on average and in most of the states considered. In India, especially in the rural areas, men perceive open defecation to be an expression of masculinity, intra-household power, health, and convenience (Coffey et al., 2014). The higher rate of latrine use among women may be initially viewed as positive, reflecting value of women's dignity and safety and indicating social inclusion. However, millions of women in rural India use the time they spend walking to the fields to be away from their in-laws, household chores, and other daily burdens and socialize with their friends or other women in the village (Routray et al., 2015; Vyas et al., 2019). While the reduction in open defecation is important, efforts must be made to avoid the reduction in women's freedom of movement that may accompany it. Further, efforts need to be made to normalize latrine use among men. Latrine use is not just for women, and use by all is necessary if positive health impacts are to be achieved.

We find that lower caste individuals are more likely to use latrines compared to higher caste individuals in our sample. This may be because socio-cultural beliefs regarding ritual purity, cleanliness, and disgust in rural areas of India can cause higher castes to not use latrines (Coffey et al., 2014). Higher castes in India perceive emptying their own latrines as extremely degrading (Coffey et al., 2017). This results in latrines with full pits being abandoned rather than emptied (Coffey et al., 2017). In order to maintain low rates of open defecation, interventions will need to target the combined and interacting barriers of caste and fear of latrine pits filling in India (Lahiri et al., 2017; Routray et al., 2015; Vyas et al., 2019). Efforts should also be made to protect the dignity of dalits, who have traditionally been employed in pit emptying (Coffey et al., 2017; Prasad and Ray, 2019, 2018).

We find no average effect of the interventions on scores of the latrine observation index. This is likely because scores increased in Odisha and decreased in Bihar and Gujarat. In other words, over time, the characteristic signs that a latrine was in use became more common in Odisha and less common in Bihar and Gujarat. The decrease in the latrine observation index scores in two states was not expected given results from selfreported latrine use. In Gujarat, different survey firms were used before and after the intervention, which may have resulted in variation in the enumerators' scoring practices. There were no consistent patterns across states in the relationship between an 
individual's demographic characteristics and the number of characteristics signs of latrine use observed in that person's household latrine.

The two measures, self-reported latrine use and the latrine observation index, each have different strengths, limitations, and interpretations. Through self-reported latrine use, we measure the behaviour of each individual separately, allowing for a more precise measure than the latrine observation index. However, unlike the latrine observation index, it is prone to reporting bias. To address this, we conducted a sensitivity analysis in which we randomly re-assigned $25 \%$ of those who reported latrine use to be true non-users. Because the intervention highlighted the social desirability of latrine use, this bias could increase more in the intervention sites than control sites, resulting in an inflation of the intervention effect estimate that would not have been addressed through our sensitivity analysis. The latrine observation index, while not prone to self-reporting and social desirability biases, can have biases related to variation in observer reliability and detection bias because observers knew treatment status. Furthermore, this approach fails to capture the known heterogeneity in behaviour within households (Vyas et al., 2019). It is not sensitive to scenarios in which only the women, sick, or elderly use the latrine. It is likely that a latrine that was regularly used by only a small portion people in a household would have the characteristic signs of use.

We acknowledge several other limitations. The use of a two-step implementation process in which nine formative evaluations were conducted and the most promising four were ultimately implemented, induces a form of publication bias. We do not know what the effects of the other five studies would have been. Therefore, our average treatment effect does not reflect the average effect that could be expected of any behavioural-science informed intervention. Rather, it reflects the average effect of these promising interventions. We hope that the positive average effects found through this approach motivate others to adopt an extended scoping process in which multiple interventions are tested and only the most promising are carried forwards. In order to respond to the variations in local context, the interventions implemented in each location were different. The strength of this approach is that it allows for the evaluation of appropriately designed and locally relevant, behavioural-science informed interventions. However, it also means that we do not know if variations in results are due to variation in local context or the design of the interventions. Finally, this work is a secondary data analysis and is prone to all the limitations of such analysis.

\section{Conclusion}

Through this meta-analysis, we show that behavioural-science informed interventions that are designed through a rigorous formative process modestly increased selfreported latrine use on average. While the individual evaluations show that some of the interventions increase self-reported latrine use, this combined analysis indicates that these impacts can be expected and are not isolated incidents. However, there was no 
impact on the household-level, latrine observation index scores. Although results appear promising, we are not able to conclusively demonstrate that these interventions increased the behaviour of latrine use.

\section{Acknowledgements}

We appreciate the time and support offered by the participants in this study. We acknowledge the contribution of each of the four teams. We therefore thank the team from Oxford Policy Management Limited whose lead principal investigator was Shruti Viswanathan, the team from the London School of Hygiene and Tropical Medicine whose lead principal investigator was Val Curtis, and the team from Swiss Federal Institute of Aquatic Science and Technology (EWAG) whose lead principal investigator was Hans-Joachim Mosler, and the team from Emory University whose lead principal investigator was Bethany Caruso. We further appreciate the work of the implementing partners of each of these teams, including the Rural Welfare Institute which was led by Pradhakar Nanda, the Indian Institute of Public Health, Gandhinagar (IIPHG) which was lead by Dileep Mavalankar, Water Aid India which was led by Arundati Muralidharan, and World Vision India which was led by Mahesh Nathan. We also acknowledge the contribution of various members of the 3ie team, including Radhika Menon and Neeta Goel. 


\section{References}

Agency, I.V., 2019. National Annual Rural. New Delhi.

Barnard, S., Routray, P., Majorin, F., Peletz, R., Boisson, S., Sinha, A., Clasen, T., 2013. Impact of Indian Total Sanitation Campaign on Latrine Coverage and Use: A Cross-Sectional Study in Orissa Three Years following Programme Implementation. PLoS One 8. https://doi.org/10.1371/journal.pone.0071438

Bottomley, C., Kirby, M.J., Lindsay, S.W., Alexander, N., 2016. Can the buck always be passed to the highest level of clustering? BMC Med. Res. Methodol. 16, 1-8. https://doi.org/10.1186/s12874-016-0127-1

Caruso, B., Sclar, G., Rotary, P., Majorin, F., Nagel, C., Clasen, T., 2019a. A clusterrandomized multi-level intervention to increase latrine use and safe disposal of child feces in rural Odisha, India: the Sundra Grama research protocol. BMC Public Health 19, 1-9.

Caruso, B., Sclar, G., Rotary, P., Nagel, C., Majorin, F., Sola, S., Koehn, W., De Shay, R., Udaipuria, S., Williams, R., 2019b. Impacts of low-cost interventions to improve latrine use and safe disposal of child faeces in rural Odisha, India. Int. Initiat. Impact Eval.

Chuahan, K., Schmidt, W., Aunger, R., Gopalan, B., Saxena, D., Yashobant, S., Patwardhan, V., Curtis, V., 2019. The 5 star toilet campaing: improving toilet use in rural Gujarat.

Coffey, D., Gupta, A., Hathi, P., Khurana, N., Spears, D., Srivastav, N., Vyas, S., 2014. Revealed Preference for Open Defecation. Econ. Polit. Wkly. 49, 43-55.

Coffey, D., Gupta, A., Hathi, P., Spears, D., Srivastav, N., Vyas, S., 2017. Understanding open defecation in rural India untouchability, pollution, and latrine pits. Econ. Polit. Wkly.

Department of Drinking Water \& Sanitaiton, 2020. Swachh Bharat Mission - Garmin [WWW Document]. URL https://swachhbharatmission.gov.in/sbmcms/index.htm

Friedrich, M., Balasundaram, T., Muralidharan, A., Raman, V., Mosler, H.-J., 2019. Promoting latrine use in rural Karnataka using the risks, attitudes, norms abilities and self-regulation (RANAS) approach. Int. Initiat. Impact Eval.

Gupta, A., Khalid, N., Deshpande, D., Hathi, P., Kapur, A., N, S., Vyas, S., Spears, D., Coffey, D., 2019. Changes in open defecation in rural North India: 2014-2018, Economic \& Political Weekly.

International Initiative for Impact Evaluation, 2020. Promoting Latrine Use in Rural India Evidence Programme [WWW Document]. URL https://www.3ieimpact.org/ourwork/water-sanitation-and-hygiene/promoting-latrine-use-rural-india-evidenceprogramme

Ioannidis, J., 2017. Next-generation systematic reviews: Prospective meta-analysis, individual-level data, networks and umbrella reviews. Br. J. Sports Med. 51, 1456- 
1458. https://doi.org/10.1136/bjsports-2017-097621

Juran, L., Adams, E.A., Prajapati, S., 2019. Purity, Pollution, and Space: Barriers to Latrine Adoption in Post-disaster India. Environ. Manage. 456-469. https://doi.org/10.1007/s00267-019-01202-6

Lahiri, S., Yegbemey, R.N., Goel, N., Mathew, L., Puri, J., 2017. Promoting latrine use in India. 3ie Scoping Paper, International Initiative for Impact Evaluation.

Models, P., Models, N., Periods, A., 2011. The Estimation of Causal Effects by Difference-in-Difference Methods By Michael Lechner. Now. https://doi.org/10.1561/0800000014

O'Reilly, K., Dhanju, R., Goel, A., 2017. Exploring "The Remote" and "The Rural" : Open Defecation and Latrine Use in Uttarakhand, India 93, 193-205. https://doi.org/10.1016/j.worlddev.2016.12.022

Prasad, C., Ray, I., 2019. When the pits fill up: (in)visible flows of waste in urban India. J. Water Sanit. Hyg. Dev. 9, 338-347. https://doi.org/10.2166/washdev.2019.153

Prasad, C., Ray, I., 2018. "When you start doing this work, it is hard to eat dal":Life and work of manyal scavengers. Econ. Polit. Wkly. LIII, 25-27.

Robitzsch, A., Grund, S., 2020. miceadds: some additional multiple imputation functions.

Routray, P., Schmidt, W., Boisson, S., Clasen, T., Jenkins, M.W., 2015. Socio-cultural and behavioural factors constraining latrine adoption in rural coastal Odisha: an exploratory qualitative study. BMC Public Health. https://doi.org/10.1186/s12889015-2206-3

Schmidt, Wolf-peter, Chauhan, K., Priya, B., Yasobant, S., Patwardhan, V., Aunger, R., Mavalankar, D., Saxena, D., Curtis, V., 2020. Cluster-randomized trial to test the effect of a behaviour change intervention on toilet use in rural India: results and mthodological considerations. BMC Public Health 20, 1-16.

Sinha, A., Nagel, C.L., Schmidt, W.P., Torondel, B., Boisson, S., Routray, P., Clasen, T.F., 2017. Assessing patterns and determinants of latrine use in rural settings: $A$ longitudinal study in Odisha, India. Int. J. Hyg. Environ. Health 220, 906-915. https://doi.org/10.1016/j.ijheh.2017.05.004

van Buuren, S., Grothuis-Oubshoorn, K., 2011. mice: Multivariate IMputation by Chain Equations in R. J. Stat. Softw. 45, 1-67.

Viswanathan, S., Saith, R., Chakraborty, A., Purty, N., Malhotra, N., Singh, P., Mitra, P., Padmanabhan, V., Datta, S., Harris, H., Gidwani, S., Williams, R., Florence, E., Daniels, S., 2019. Improving households' attitudes and behaviours to increase toilet use in Bihar, India. Int. Initiat. Impact Eval.

Vyas, S., Srivastav, N., Mary, D., Goel, N., Srinivasan, S., Tannirkulam, A., Ban, R., Spears, D., Coffey, D., 2019. Measuring open defecation in India using survey questions: Evidence from a randomised survey experiment. BMJ Open 9, 1-9. 
https://doi.org/10.1136/bmjopen-2019-030152

WHO / UNICEF, 2012. Water sanitation hygiene [WWW Document]. Fast facts. URL https://www.who.int/water_sanitation_health/monitoring/jmp2012/fast_facts/en/ 


\section{Supplemental materials 1}

Description of interventions and study design

Bihar intervention - Improving households' attitudes and behaviours to increase toilet use (HABIT)

Conducted by Oxford Policy Management

Based on nudge theory, this team attempted to change attitudes and practices around toilet use. They used a combination of household and community level meetings in which they encouraged latrine use through posters, a French drain demonstration, and a card game. They also used tools to encourage commitment to the adoption of the behaviour such a pledge and a calendar for a reminder. The intervention had high levels of coverage and almost all households received the intervention in the treatment group.

The team had extensive quality control measures for quantitative and qualitative surveys which included: field supervision and quality assurance; daily data consistency checks; data back checks; daily debriefs and revisit interviews where necessary.

86 villages were selected where World Vision has presence in Bihar. 43 were randomly assigned to treatment and rest to control. From the list of villages, 44 treatment wards and 44 control wards were selected by Probability proportion to size sampling. A sample of 12 households were selected randomly from a list of eligible households in each ward.

\section{Karnataka intervention - RANAS}

Conducted by the Swiss Federal Institute of Aquatic Science and Technology (EWAG) This intervention addressed perceived risks, attitudes, norms, abilities, and selfregulation (RANAS) through community meetings and household visits over a span of 7-8 days. There were two community sessions and two household visits, with the two household visits between the two community sessions. They also conducted a meeting at anganwadi centers to promote the safe disposal of child feces. During this intervention, a supportive phone call was used to remind individuals to use the latrine. The intervention reach was assessed at $83 \%$ in the treated villages.

The data collection team comprised on 21 members, which included 7 supervisors and 14 enumerators. Each supervisor was responsible for 2 to 4 enumerators. Two tools were used- a structured quantitative face-to-face questionnaire and a spot-check observations of household latrine. The supervisors conducted back-checks. The data was collected on a CAPI platform and data was regularly checked for consistency and quality.

250 villages of 1071 villages were eligible (More than 30\% latrine coverage). 120 villages were randomly selected. Out of the households with functioning latrines, randomly select 2328 households for baseline. In the endline 10 villages were dropped as latrine coverage was 99\%. The final sample was 2169 households from 110 villages.

Gujarat intervention - The 5 Star Toilet Campaign

Conducted by the London School of Hygiene and Tropical Medicine 
Based on the theory and process of behaviour centered design, this team developed a social marketing campaign to promote latrines as smart and modern. The intervention used community events including a World of Toilets activity, a virtual reality tool, skits and films, a toilet board, songs, a composting guessing game, a life sized demonstration pit, certificates and stickers, and a SMART wi-fi network. All participants in the target population could have been exposed to treatment.

The two tools in the data collection process were administered though CAPI tools by trained enumerators. There was a span of 5-7 days in between the administration of two tools. The quality of data collection was closely monitored by supervisors.

94 villages/clusters were randomly selected from 137 eligible (latrine coverage $>70 \%$ ) clusters. 47 villages were randomly assigned to treatment and the rest to control. 10 Households from each village were randomly selected for the baseline survey. There households were not included in endline. In the endline 30 other households were randomly selected. All members of the household were interviewed for the main survey and two members for the physical activity survey.

\section{Odisha intervention - Sundra Grama}

Conducted by Emory University

Emory addressed perceptions of risk, ability, social norms, motivation, physical opportunity, and self-regulation in order to promote latrine use. They used community meetings and household visits. A special meeting for mothers was used to promote the safe disposal of child feces. Pallas and transect walks were used to promote latrine use in an engaging manner. Paintings and positive deviant recognition attempted to change perceptions of social norms. Minor latrine repairs were done as well.

The intervention reach was assessed at $85 \%$ in the treated villages.

A team of data collectors supervised by a field manager and a PI conducted the quantitative data collection. Periodic data quality checks were conducted. Data quality checks were performed periodically. The team put into action effective strategies to ameliorate different biases such as social desirability bias.

To select the villages, the research team carried out a rapid assessment by visiting 282 villages across 3 blocks. From this the team 72 villages based on village size, latrine coverage, ODF status. 6 villages were purposively selected for qualitative research and remaining 66 for quantitative survey. 33 were randomly assigned in treatment and rest in control. In total there were 3978 households at baseline and 4280 households at endline. 


\section{Supplemental materials 2}

Latrine observation questions

1. Is the latrine being used for some other purpose?

2. Is the squatting pan clogged with leaves / dirt / other materials?

3. Water container, like lota, mug, or coke bottle (for washing after defecation) in the latrine?

4. Slippers outside or inside the latrine?

5. Are there supplies to clean the latrine pan (i.e. toilet brush, cleaning fluid like Harpic)?

6. According to your (enumerator's) judgement, does the latrine look like it is likely being used?

Latrine use question

For every household member five or older, as part of a household roster (where household is defined as living under this roof): the last time \{NAME\} defecated, did \{NAME\} defecate in the open or use the latrine?

\section{Supplemental materials 3: Quantitative synthesis report pre- analysis plan}

\section{Overview}

The Sustainable Development Goals (SDG 6.2) target the ending of open defecation by 2030. $60 \%$ of those defecating in the open reside in India. In an effort to eradicate open defecation by October 2019, the Indian government launched its flagship program the Swachh Bharat Mission (Clean India Mission; SBM) in 2014. Given that most Indian households did not have access to toilets, the primary focus of the program was the large scale construction of toilets. Yet, several studies suggest that access to a toilet does not necessarily imply use.

In 2016, the International Initiative for Impact Evaluation (3ie) launched a thematic window to supplement the work being done under the SBM. 3ie's Promoting Latrine Use in Rural India thematic window used insights from behavioural science to generate evidence on the design and implementation of context-specific, low-cost interventions that can be implemented and scaled-up with the resources available through the Government of India's Swachh Bharat Mission (Clean India Mission; SBM) and/or any voluntary household contributions. During the three-month formative phase of the window, nine teams tested interventions in eight Indian states to develop contextspecific, mature interventions. Based on these findings, 3ie funded four impact evaluations of interventions to promote latrine use in rural India. The four teams conducted impact evaluation studies over 18 months in the states of Bihar, Odisha, 
Gujarat and Karnataka. Additional information on the teams and their interventions can be viewed here.

We aim to synthesize quantitative findings across these studies. Because we have access to individual level data from this set of prospective studies, each of which used the same questions, our analysis is novel in that it will be a prospective, individual-level meta-analysis (Ioannidis 2017). Such an analysis is considered the gold standard of meta-analysis. Although the specific interventions are different, they all had similar, twostage designs: a three-month formative window in which specific, behavioural barriers to latrine use were identified within a target population, and then a behavioural science informed intervention to support latrine use among their target populations was implemented. Because the impact evaluations of these interventions use a pre-specified and similar set of questions, this analysis can evaluate the impact of a two-stage, behavioural science approach on improving latrine use in rural India among the identified target populations. This quantitative pre-analysis plan provides the details for this synthesis.

\section{Sample}

At baseline, the sampling across the studies began with the selection of districts. Three studies operated in one district each, while the Bihar study identified six districts. The districts were usually selected due to the implementing partners' prior experience of working in these areas. Eligibility at the village level was determined on factors such as current latrine coverage rates (to ensure there were adequate households with latrines), village size, and non-Open Defecation Free (ODF) status (Odisha). Eligible villages were then randomly assigned to treatment or control. The selection of households varied by team. Three of the teams re-interviewed the same households at endline, while Gujarat interviewed a different set of households. Study descriptions are presented in table 1.

\section{Data aggregation and common variables across studies}

All four impact evaluations focus on latrine use (in rural India, among household members who are age $6+$ ) as the primary outcome. In the case of children aged 5 and below, the safe disposal of feces is the primary outcome. The data used for this synthesis was extracted from four impact evaluations datasets. We merged these datasets to construct an aggregated dataset. This is possible because, from the outset, a set of standardized questions was developed in collaboration with the four teams. All four teams were free to include additional questions. The list of standardized questions is presented below in table 2 . 
Table 1: Baseline study samples and eligibility across projects

\begin{tabular}{|c|c|c|c|c|c|}
\hline State & Eligibility criteria & Districts & Villages & $\begin{array}{l}\text { Number of } \\
\text { individuals sampled } \\
\text { at baseline }\end{array}$ & $\begin{array}{l}\text { Number of } \\
\text { individuals sampled } \\
\text { at endline }\end{array}$ \\
\hline Bihar & $\begin{array}{l}\text { Households with } \\
\text { functional twin pit latrines }\end{array}$ & $\begin{array}{l}6 \text { districts } \\
\text { (Jamui, } \\
\text { Khagaria, } \\
\text { Madhepura, } \\
\text { Nalanda, } \\
\text { Nawada } \\
\text { and } \\
\text { Saharsa) }\end{array}$ & $\begin{array}{l}86 \text { quantitative } \\
\text { villages } \\
6 \text { qualitative } \\
\text { villages } \\
\text { ( } 28 \text { ODF villages) }\end{array}$ & 6,158 & 6,046 \\
\hline Gujarat & $\begin{array}{l}\text { Households with } \\
\text { functional government or } \\
\text { contractor built latrines }\end{array}$ & $\begin{array}{l}1 \text { district } \\
\text { (Bhavnagar) }\end{array}$ & $\begin{array}{l}90 \text { quantitative } \\
\text { villages } \\
4 \text { qualitative } \\
\text { villages } \\
\text { (All ODF villages) }\end{array}$ & $\mathrm{NA}^{1}$ & 13,960 \\
\hline Karnataka & $\begin{array}{l}\text { Households with } \\
\text { functional latrines }\end{array}$ & $\begin{array}{l}1 \text { district } \\
\text { (Raichur) }\end{array}$ & $\begin{array}{l}120 \text { quantitative } \\
\text { and qualitative } \\
\text { villages } \\
\text { (No ODF villages) }\end{array}$ & 10,793 & 10,656 \\
\hline Odisha & Households with latrines & $\begin{array}{l}1 \text { district } \\
\text { (Puri) }\end{array}$ & $\begin{array}{l}66 \text { quantitative } \\
\text { villages } \\
6 \text { qualitative } \\
\text { villages } \\
\text { (6 ODF villages) }\end{array}$ & 16,827 & 16,827 \\
\hline
\end{tabular}


Table 2: Standardized Questions

\begin{tabular}{|c|c|c|}
\hline & & Variable Name \\
\hline & \multicolumn{2}{|l|}{ Latrine Observation } \\
\hline 1 & $\begin{array}{l}\text { Is the latrine being used for some other purpose? [Other } \\
\text { purpose indicates non-use.] }\end{array}$ & latrine_use_other \\
\hline 2 & $\begin{array}{l}\text { Is the squatting pan clogged with leaves/dirt/other } \\
\text { materials? [Leaves/dirt/other materials indicate non- } \\
\text { use.] }\end{array}$ & latrine_nonuse \\
\hline 3 & $\begin{array}{l}\text { Water container, like lota, mug, or coke bottle, (for } \\
\text { washing after defecation) in the latrine? [Water } \\
\text { container indicates use.] }\end{array}$ & latrine_use_water \\
\hline 4 & $\begin{array}{l}\text { Slippers outside or inside the latrine? [Slippers indicate } \\
\text { use.] }\end{array}$ & latrine_use_slipper \\
\hline 5 & $\begin{array}{l}\text { Are there supplies to clean the latrine pan (i.e. toilet } \\
\text { brush, cleaning fluid like Harpic)?[Cleaning supplies } \\
\text { indicate use.] }\end{array}$ & latrine_use_clean \\
\hline 6 & $\begin{array}{l}\text { According to your (enumerator's) judgment, does the } \\
\text { latrine look like it is likely being used? }\end{array}$ & latrine_use_obs \\
\hline & Latrine Use & \\
\hline 7 & $\begin{array}{l}\text { For every household member five or older, as part of a } \\
\text { household roster (where household is defined as living } \\
\text { under this roof): "The last time [NAME] defecated, did } \\
\text { [NAME] defecate in the open or use the latrine?" }\end{array}$ & defecate_where_adult \\
\hline 8 & $\begin{array}{l}\text { For children younger than five: The last time [NAME of } \\
\text { child under 5] defecated, where did [NAME of child } \\
\text { under 5] defecate? }\end{array}$ & defecate_where_child \\
\hline 9 & $\begin{array}{l}\text { (For children younger than } 5 \text { and if child went } \\
\text { somewhere other than "In latrine"): What was done to } \\
\text { dispose of the stools? }\end{array}$ & defecate_child_dispose \\
\hline & Household Latrine & \\
\hline 10 & For all households: & \\
\hline & $\begin{array}{l}\text { a. Have you received any money or materials from the } \\
\text { government or an NGO to construct a latrine? }\end{array}$ & latrine_construct_receipt \\
\hline & $\begin{array}{l}\text { b. (If materials in a) Did the government or NGO give } \\
\text { you materials, or did it construct the latrine for you? }\end{array}$ & materials_provide_who \\
\hline 11 & For households that have a latrine: & \\
\hline & $\begin{array}{l}\text { a. How long ago was construction completed on the } \\
\text { latrine? }\end{array}$ & latrine_construct_time \\
\hline & $\begin{array}{l}\text { b. Does the latrine have one pit, two pits, or a septic } \\
\text { tank? }\end{array}$ & latrine_pit \\
\hline
\end{tabular}




\begin{tabular}{|l|l|l|}
$\begin{array}{l}\text { c. How big is the pit? [Instructions: For pits that are } \\
\text { cylindrical, either record the number of rings if rings } \\
\text { have been used, or record the diameter and depth. If } \\
\text { the latrine has a septic tank, record the dimensions of } \\
\text { the tank under first pit and leave second pit blank.] }\end{array}$ & \\
\hline d. Has the pit of your latrine ever filled up? & latrine_pit_size \\
\hline $\begin{array}{l}\text { e. (If yes to d) What did you do with the pit after it filled } \\
\text { up? Did you get it emptied, did you dig a new pit or start } \\
\text { using second pit, or did some people stop using? }\end{array}$ & latrine_pit_fill \\
\hline f. (If emptied in e) How was it emptied? & latrine_pit_empty \\
\hline
\end{tabular}

We identified the following primary and secondary outcomes, and list of indicators. We have extracted information on these outcomes and indicators from the four studies.

Table 3 lists all the indicators and outcomes.

Table 3: Outcomes (definitions list) and key indicators

\begin{tabular}{|c|c|}
\hline Outcomes & $\begin{array}{l}\text { Type of } \\
\text { Outcome }\end{array}$ \\
\hline \multicolumn{2}{|l|}{ Primary } \\
\hline Latrine use ${ }^{2}$ & I \\
\hline Safe disposal of child faeces ${ }^{3}$ & I \\
\hline Observed latrine use & $\mathrm{H}$ \\
\hline \multicolumn{2}{|l|}{ Secondary } \\
\hline $\begin{array}{l}\text { Correlation between latrine observation and } \\
\text { reported use }\end{array}$ & $\mathrm{H}$ \\
\hline Predictors of use & $\begin{array}{l}\text { Type of } \\
\text { Indicator }\end{array}$ \\
\hline Type of latrine ${ }^{4}$ & $\mathrm{H}$ \\
\hline Size of pit & $\mathrm{H}$ \\
\hline Sources of funding & $\mathrm{H}$ \\
\hline Latrine age & $\mathrm{H}$ \\
\hline Education of the head of household 5 & I \\
\hline Caste & $\mathrm{H}, \mathrm{I}$ \\
\hline Sex & I \\
\hline Household Size & $\mathrm{H}$ \\
\hline $\begin{array}{l}\text { Note: "I" is Individual level indicator, " } \mathrm{H} \text { " is } \\
\text { household level indicator }\end{array}$ & \\
\hline
\end{tabular}

\footnotetext{
2 Self-reported latrine use among individuals over five years of age

${ }^{3}$ Safe disposal: faeces disposed or rinsed in latrine

${ }^{4}$ The type of latrine differs by team. For example, the Bihar team only considered twin pit latrines.

${ }^{5}$ For Odisha we can only disaggregate by education at the household level, as we know the household head's education.
} 


\section{Hypotheses}

The goal of this analysis is to quantify the impact of the approach taken by 3ie to address latrine use in rural India among the identified target populations. This approach involved a two-stage intervention design in which local, behavioural barriers were first identified through a three-month formative phase and then behavioural science informed projects were implemented to address these barriers. By testing the following hypotheses, we will demonstrate the extent of the impact of the interventions on latrine use in rural India among the identified target population. The hypotheses are first presented here, then details on the analytical approach are presented in the following section.

Primary outcome 1: Self-reported latrine use among individuals older than five years of age.

There will be a larger increase in self-reported latrine use rates in intervention sites than control sites

Primary outcome 2: Observed latrine use as measured through an observation index reflecting if the latrine appears to be in use There will be a larger increase in household level latrine use rates measured through observation in intervention sites than control sites

Primary outcome 3: Safe disposal child faeces There will be a larger increase in the rates of the safe disposal of child faeces in intervention sites than control sites

Secondary outcome 1: Correlation between self-reported latrine use and observed latrine use Households whose latrines appear to be in use will be more likely to have at least one member who reports latrine use than households whose latrine does not appear to be in use

\section{Predictors of latrine use}

\section{Household demographics}

Across intervention and control sites, individuals in households with more people will be less likely to use latrines

Across intervention and control sites, individuals in households whose household heads have lower education levels will be less likely to use latrines 


\section{Individual demographics}

Across intervention and control sites, men will be less likely to use latrines than women Across intervention and control sites, middle-aged individuals will be most likely to use latrine

Across intervention and control sites, individual-level latrine use rates will be lower among scheduled castes and scheduled tribes

\section{Latrine characteristics}

Across intervention and control sites, individuals in households with twin pit latrines will be more likely to use latrines

Across intervention and control sites, individuals in households with smaller latrine pits will be less likely to use latrines Across intervention and control sites, individuals in households that contributed their own resources to latrine construction will be more likely to use latrines Across intervention and control sites, individuals in households that use older latrines will be less likely to use the latrine

\section{Analysis}

\section{Latrine Observation Index}

This index will be created using the latrine spot check observations of toilets conducted by the researchers in different states. The index will range between 0 and 1 , with 0 indicating none of the spot check items suggest latrine use and 1 indicating all of the spot check item suggests use.

\section{Index construction}

Item selection: The items for the index are a component of the standardized questions. There are 6 items in the latrine observation index. Each of these items measure latrine usage at the household level. The items are listed in table 1 under Latrine Observation.

\section{Empirical relationships}

We will inspect the correlation between the items before inclusion. From our review of the baseline data, the items are positively correlated across data from all four teams

\section{Index scoring}

All the items are coded "0" and " 1 ", with " 0 " indicating No and " 1 " indicating Yes. All the responses (six items) are added together for each household to arrive at household level score of observed latrine use. The score is standardized to interpret it as observed latrine use. We use the following mathematical model to standardize the scores. 


$$
\text { Latrine observation index }=\frac{\text { Score }_{i}-\text { Score }_{\text {min }}}{\text { Score }_{\max }-\text { Score }_{\text {min }}}
$$

Because item six is likely to be affected by the scores of the other items and, therefore, is not an independent measure, a sensitivity analysis will be conducted in which this item is dropped from the development of the latrine observation index.

\section{Sample descriptions}

See attached table 1 and supplemental table 1 for the table shell that will be filled in and presented.

\section{Meta-analysis}

We propose an individual level meta-analysis in which the data from the individual participants from each of the studies will be analyzed. Individual-level and prospective meta-analyses are now being viewed as gold standards. We are able to achieve this gold standard, but a few challenges still exist to data harmonization and interpretation. These are described with potential solutions here. After, the proposed analyses are outlined.

\section{Expected challenges and solutions}

\section{Variation in study context and design}

Challenge: Each study had slightly different criteria for inclusion and exclusion. These differences range from the identification of eligible households to the identification of eligible villages. Furthermore, India is a highly diverse country. Each state is different, with a unique culture, history, socioeconomic profile, and set of barriers to latrine use. Therefore, what is effective for one population or state may not have the same effect in another. If treatment effects vary by factors related to the different inclusion criteria or state, it would be inappropriate to calculate a single treatment effect across the four studies.

Solution: This is the reason that the same intervention was not implemented in all four studies. We acknowledge that what works for one population in one area may not work in another. We are testing if this two-stage, behaviour science informed intervention approach can be effective across different contexts within identified target populations; therefore, these differences in context are necessary to answer the question. Because the goal of this analysis is to understand if the interventions are effective within their target populations, differences in target population are acceptable.

Missing baseline data

Challenge: A different set of households was interviewed at baseline and endline in Gujarat. In the other three studies, the same households were interviewed at both times. 
Solution: Data for latrine observation variables will be imputed for baseline values using the available baseline data. This is not possible for latrine use as the question was not asked at the individual level.

\section{Potential for selection bias}

Challenge: 3ie originally commissioned nine formative studies and then only funded the four that were believed the most likely to have an impact. As such, these studies are likely to have somewhat larger effects than if studies had been chosen at random.

Solution: There is no statistical approach that will allow us to correct this selection bias. It is simply a limitation of our study. We must acknowledge that our summary effect size may overestimate the effects expected from any randomly chosen behaviour change study.

\section{Proposed analyses}

We will estimate the effect size of each of the following analyses individually and then calculate a combined effect, reflecting the meta-analysis. These effects will be visually reflected in a forest plot. Based on analysis from the Measurement Study, also commissioned by 3ie but using a different sample of households, the following variables were significantly related to latrine use at baseline and endline in at least one state and will therefore be included as controls: sex, age, education, occupation, caste, latrine age, latrine type, and latrine financing. Standard errors will be clustered at the state level (Bottomley et al 2016). Data analysis will be conducted in STATA and R. For the sake of clarity, each hypothesis is re-stated here, and the proposed analysis is outlined below.

Primary outcome 1: Self-reported latrine use

There will be a larger increase in reported latrine use rates in intervention sites than control sites

Analysis: Examine the difference in latrine use between intervention and control arms at endline.

Primary outcome 2: Observed latrine use

There will be a larger increase in latrine use rates measured through observation in intervention sites than control sites

Analysis: Difference-in-difference analysis examining the change in latrine use rates as measured through observation between intervention and control sites

Primary outcome 3: Safe disposal of child faeces

There will be a larger increase in the rates of the safe disposal of child faeces in intervention sites than control sites 
Analysis: Difference-in-difference examining the change in the rates of safe disposal of child faeces between intervention and control sites

Secondary outcome 1: Correlation between self-reported latrine use and observed latrine use

Households whose latrines appear to be in use will be more likely to have at least one member who reports latrine use than households whose latrine does not appear to be in use

Analysis: Logit comparing the rates of latrine use measured through observation among households in which at least one person self-reports latrine use at endline

\section{Predictors of latrine use}

Household demographics

Across intervention and control sites, individuals in households with more people will be less likely to use latrines

Analysis: Logit considering the individual-level latrine use rates as a function of the continuous measure of the number of individuals in the household Across intervention and control sites, individuals in households whose household heads have lower education levels will be less likely to use latrines

Analysis: Logit considering the individual-level latrine use rates as a function of the education level of the head of household

\section{Individual demographics}

Across intervention and control sites, men will be less likely to use latrines than women Analysis: Logit considering the individual-level latrine use rates as a function of sex

Across intervention and control sites, middle-aged individuals will be most likely to use latrine

Analysis: Logit considering the individual-level latrine use rates as a function of age and age ${ }^{2}$

Across intervention and control sites, individual-level latrine use rates will be lower among scheduled castes and scheduled tribes

Analysis: Logit considering the individual-level latrine use rates as a function of caste

\section{Latrine characteristics}

Across intervention and control sites, individuals in households with twin pit latrines will be more likely to use latrines

Analysis: Logit considering the individual-level use rates as a function of latrine type 
Across intervention and control sites, individuals in households with smaller latrine pits will be less likely to use latrines

Analysis: Logit considering the individual-level use rates as a function of latrine pit size

Across intervention and control sites, individuals in households that contributed their own resources to latrine construction will be more likely to use latrines

Analysis: Logit considering the individual-level use rates as a function of reported self-funding of latrine construction

\section{References}

Bottomley, C., \& et.al. (2016). Can the buck always be passed to the highest level of clustering? BMC medical research methodology.

Garn, J. V., Sclar, G. D., Freeman, M. C., Penakalapati, G., Alexander, K. T., Brooks, P., \& Clasen, T. F. (2017). The impact of sanitation interventions on latrine coverage and latrine use: A systematic review and meta-analysis. International journal of hygiene and environmental health, 220(2), 329-340.

Ioannidis, John. (2017) Next-generation systematic reviews: prospective meta-analysis, individual-level data, networks and umbrella reviews. British Medical Journal, 51(20), 1456-1458.

N.A. 2014. Gender in Food and Nutrition Security Programming Conducting a gender analysis for programme design This course is funded by the European Union through the EU-FAO Improved Global Governance for Hunger Reduction Programme, FAO accessed here http://www.fao.org/elearning/Course/FG/en/pdf/1348 text only 1348.pdf Rosalie Norem. 2001. Intermediate Level Handbook SEAGA Socio-Economic and Gender Analysis Programme, Prepared by in collaboration with the Socio-economic and Gender Analysis (SEAGA) Programme, Gender and Development Service, FAO 